\title{
A nucleostemin family GTPase, NS3, acts in serotonergic neurons to regulate insulin signaling and control body size
}

\author{
Daniel D. Kaplan, ${ }^{1,2}$ Gregor Zimmermann, ${ }^{1}$ Kaye Suyama, ${ }^{1}$ Tobias Meyer, ${ }^{2}$ and Matthew P. Scott ${ }^{1,3}$ \\ ${ }^{1}$ Departments of Developmental Biology, Genetics, and Bioengineering, Howard Hughes Medical Institute, Stanford \\ University School of Medicine, Stanford, California, 94305, USA; ${ }^{2}$ Department of Chemical and Systems Biology, Stanford \\ University School of Medicine, Stanford, California, 94305, USA
}

Growth and body size are regulated by the CNS, integrating the genetic developmental program with assessments of an animal's current energy state and environmental conditions. CNS decisions are transmitted to all cells of the animal by insulin/insulin-like signals. The molecular biology of the CNS growth control system has remained, for the most part, elusive. Here we identify NS3, a Drosophila nucleostemin family GTPase, as a powerful regulator of body size. ns3 mutants reach $<60 \%$ of normal size and have fewer and smaller cells, but exhibit normal body proportions. NS3 does not act cell-autonomously, but instead acts at a distance to control growth. Rescue experiments were performed by expressing wild-type ns 3 in many different cells of ns3 mutants. Restoring NS3 to only 106 serotonergic neurons rescued global growth defects. These neurons are closely apposed with those of insulin-producing neurons, suggesting possible communication between the two neuronal systems. In the brains of ns 3 mutants, excess serotonin and insulin accumulate, while peripheral insulin pathway activation is low. Peripheral insulin pathway activation rescues the growth defects of ns3 mutants. The findings suggest that NS3 acts in serotonergic neurons to regulate insulin signaling and thus exert global growth control.

[Keywords: Nucleostemin; serotonin; insulin; neurons; growth; size]

Supplemental material is available at http://www.genesdev.org.

Received March 6, 2008; revised version accepted May 23, 2008.

The overall size of an animal is determined by the number of cell divisions, the rate of destruction of cells, and the average size of cells (Conlon and Raff 1999; Edgar 2006). With some species, size is a relatively rigid outcome of the genetically controlled program of development. Other species, such as many fish and trees, can continue to grow through much of their lives. In both cases growth is influenced by continuing assessment of the energy state of the growing organism and the availability of nutrients, interpreted in the context of the organism's genetic program. The cell-intrinsic machinery that specifies whether or not a cell should divide and how large a cell should grow has been unveiled in elegant molecular detail (Conlon and Raff 1999; Edgar 2006). Much remains to be learned about the cell-extrinsic mechanisms that coordinate the growth behaviors of individual cells. Global cell-extrinsic controls are needed to ensure that a properly proportioned animal is produced, with a size suited to its environment and genetic program.

${ }^{3}$ Corresponding author.

E-MAIL mscott@stanford.edu; FAX (650) 725-2952.

Article is online at http://www.genesdev.org/cgi/doi/10.1101/gad.1670508.
Whole-animal growth control can be envisioned as having three components that operate in concert: (1) sensory and homeostatic inputs, (2) processing within the CNS, and (3) instructive outputs to the periphery. Sensory inputs to the CNS provide information regarding the energy status of the organism and the available nutrient status of the environment. The CNS assesses this information in light of the genetic program and instincts about future energy requirements; e.g., for growth, reproduction, migration, and hibernation. The CNS converts the processed information into output signals that alter feeding behavior and spread through the body to coordinate growth. The transmitted output signals instruct cells in peripheral tissues to grow, to cease growth, or to die, by influencing cell-intrinsic programs. Recent studies have shed light on the input and output signals involved in growth control (1 and 3). Much less is known about the central processing mechanisms (2).

A great deal of elegant work has identified signaling mechanisms by which energy states are sensed and the information is relayed to the CNS (Morton et al. 2006; Melcher et al. 2007). In mammals, dietary free fatty acids can act on the anterior pituitary gland to inhibit growth hormone secretion (Dieguez and Casanueva 1995). In 
Drosophila, the fat body, a major metabolic organ that performs the combined functions of the liver and adipocytes of mammals, has been established as an important source of nutrient sensing. In response to high levels of amino acids in the fat body, this organ is thought to produce an as-yet-unknown signal that acts in the brain to suppress food intake (Zinke et al. 1999). Amino acid starvation results in strongly suppressed production of a fat body-derived glycoprotein known as Drosophila acidlabile subunit (dALS) (Colombani et al. 2003). When dALS is produced, it binds to and regulates the bioavailability of Drosophila insulin-like peptides (DILPs) secreted by the neurosecretory cells of the brain (Arquier et al. 2008), thus connecting sensed amino acid levels to the activity of a critical growth signal.

The Drosophila insulin system controls cell-intrinsic growth programs throughout the body. DILPs are secreted when nutrients are plentiful to stimulate growth of target tissues (Chen et al. 1996; Oldham and Hafen 2003; Edgar 2006). DILPs are produced primarily by insulin-producing cells (IPCs) (Ikeya et al. 2002; Rulifson et al. 2002), groups of seven neuroendocrine cells located in each of the two brain lobes, and then enter the circulation. The DILPs interact with insulin receptor tyrosine kinases (InRs) on target cells (Chen et al. 1996), activating a signaling cascade that ultimately stimulates growth through an increase in protein synthesis (Chen et al. 1996; Oldham and Hafen 2003; Edgar 2006). Disruption of the insulin signaling pathway through either genetic ablation of the IPCs (Ikeya et al. 2002; Rulifson et al. 2002) or mutation of intracellular components of the pathway (Leevers et al. 1996; Bohni et al. 1999; Verdu et al. 1999) leads to major developmental delay, growth retardation, and reduced adult size.

Identifying the mechanisms operating within the CNS that serve to integrate environmental, nutritional, and physiological information to direct proper growth responses will require identifying components of these pathways and the neurons in which they act. Here we identify NS3, a nucleostemin-family GTPase, as a powerful regulator of body size. Nucleostemins were discovered (Tsai and McKay 2002) as genes highly expressed in human stem cells compared with differentiated cells, and their expression has been linked to certain types of cancer. NS3 is related to a yeast protein, Lsg1p, which functions in ribosome biogenesis; a role that is essential for growth of yeast cells (Kallstrom et al. 2003; Hedges et al. 2005). Surprisingly, we find that Drosophila NS3 is not required for growth in most cells, but rather acts specifically within serotonergic neurons to regulate insulin signaling and exert global control over cell size and number.

\section{Results}

\section{Identification of the Drosophila nucleostemin family}

We performed an RNA interference screen for novel developmental regulators, in which dsRNAs targeting uncharacterized Drosophila genes were injected into em- bryos. Progression through embryogenesis and hatching into first instar larvae were analyzed. In this screen (Zimmermann et al. 2006), we identified the gene CG3983 as being essential for embryonic development. CG3983 is the Drosophila gene most closely related to the human gene NUCLEOSTEMIN (NS or GNL3), which encodes a nucleolar GTPase that regulates cell proliferation and cell fate (Tsai and McKay 2002). CG3983 is one of four closely related Drosophila genes, which together constitute a nucleostemin family (Supplemental Fig. S1A). Based upon sequence similarity, we are designating CG3983 as Drosophila Nucleostemin 1 (NS1). The other members of the family, in order of increasing divergence from human NS, we are designating NS2 (CG6501), NS3 (CG14788), and NS4 (CG9320). These proteins share a common domain structure (Fig. 1A; Supplemental Fig. $\mathrm{S} 1 \mathrm{~A})$, consisting of a basic domain at their $\mathrm{N}$ termini, coiled-coil domains, GTP-binding motifs, a putative RNA-binding domain, and an acidic domain near their $\mathrm{C}$ termini. The GTP-binding motifs are circularly permuted compared with their arrangement in most GTPases. The G1-G4-binding domains are ordered G4-G1G2-G3 from the $\mathrm{N}$ to $\mathrm{C}$ termini, rather than the canonical G1-G2-G3-G4 orientation observed in Ras family GTPases (Bourne et al. 1991). We found that NS1 and NS2 expressed as YFP-fusion proteins in cultured Drosophila S2 cells share the nucleolar localization of human NS, but NS3 has a punctate, cytoplasmic distribution (Supplemental Fig. S1B). The same punctate, mostly cytoplasmic localization pattern of NS3-YFP was observed when it was produced in larval salivary glands (Supplemental Fig. S1C) and neurons (Supplemental Fig. S1D). In experiments described below we confirmed that the NS3-YFP fusion protein is functional. The N-terminal basic domain has been shown to be required for the nucleolar localization of human NS (Tsai and McKay 2002). The number and arrangement of $\mathrm{N}$-terminal basic residues varies between members of the Drosophila Nucleostemin family and is lowest in NS3, which may explain its distinct localization.

To evaluate the roles of Drosophila Nucleostemins in development, we microinjected Drosophila embryos with dsRNAs targeting $n s 1, n s 2$, or $n s 3$, and analyzed the effect of a homozygous mutation in $n s 4$. We found that dsRNAs targeting either $n s 1$ or $n s 2$ impaired embryonic development, resulting in a profoundly reduced rate of larval hatching (Supplemental Fig. S1E). Animals homozygous for a P-element insertion that disrupts the ns4 gene proceeded through normal embryonic and larval development and emerged as healthy adult flies, indicating that ns4 is not likely essential for Drosophila development (Supplemental Fig. S2). Embryos that had been injected with dsRNA specific for ns3 progressed through embryogenesis and hatched into first instar larvae in a manner indistinguishable from controls (Supplemental Fig. S1E). Following hatching, their growth was severely impaired compared with control animals (Supplemental Fig. S1F). On this basis and its distinct localization, NS3 seemed particularly interesting, so we focused on how it controls growth and body size. 
A

NS3 (CG14788)

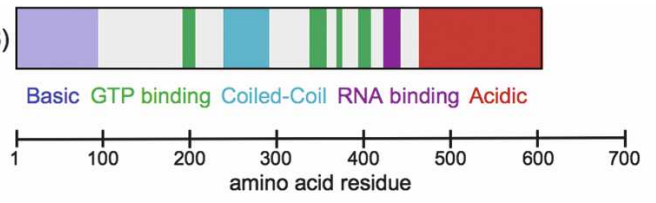

B
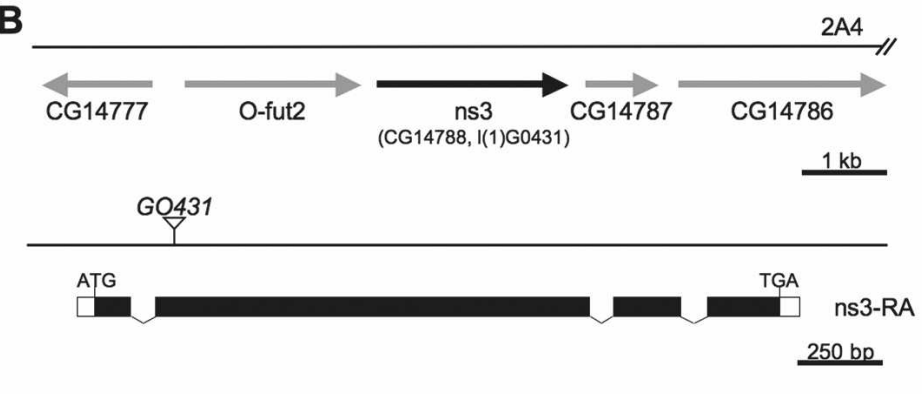

C
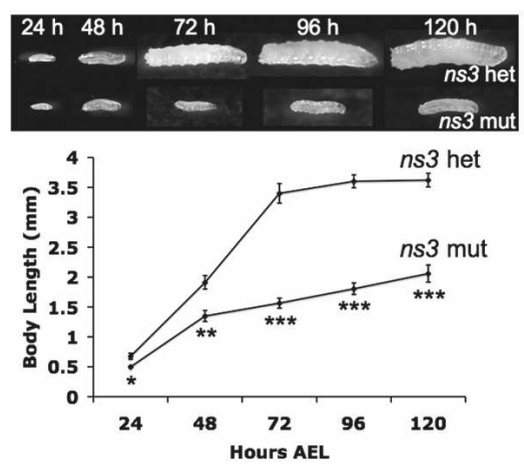

E

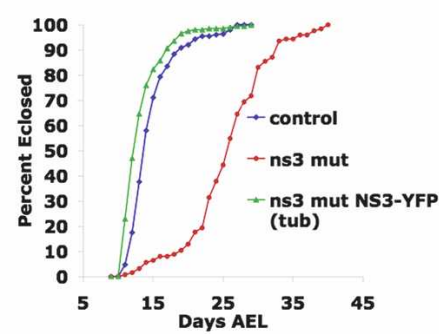

F 200

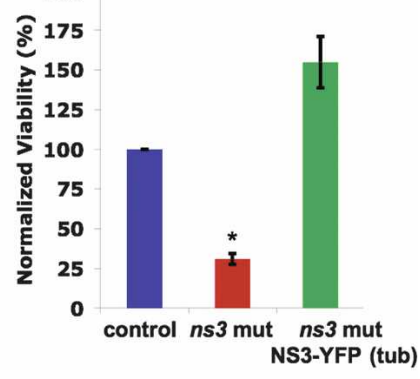

G

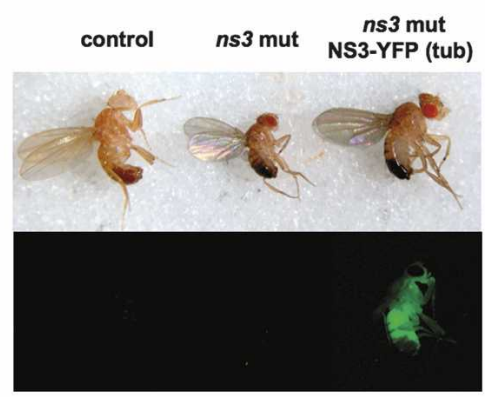

D

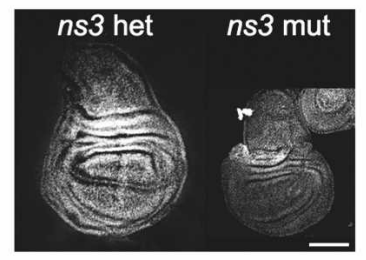

Figure 1. Growth phenotype of ns3 mutant. $(A)$ Domain structure of NS3. (B) ns3 genomic locus (top) and exon/ intron organization showing P-element insertion (bottom). (C) Mutation of ns3 retards larval growth. Body length was compared between ns3 heterozygous (het) and mutant (mut) larvae. $\left(^{*}\right) P=0.0031$, $\left.^{(\star *}\right) P=0.0011$, $\left(^{\star \star \star}\right) P<0.0001$ for $n s 3$ het versus mut $(n=10$ per condition per time point). $(D)$ Wing imaginal discs are smaller in ns3 mutant larvae. $(E, F)$ ns3 mutant animals exhibit a profound developmental delay and reduced viability that can be rescued by expression of NS3-YFP using a Tubulin-Gal4 driver (tub). $\left({ }^{\star}\right) \mathrm{P}<0.0001$ for $n s 3 \mathrm{mu}-$ tant $(n=146)$ compared with control $(n=388)$ or $n s 3$ mutant + NS3-YFP (tub) $(n=221) .(G) n s 3$ mutants are dramatically reduced in size and the size defect can be rescued by ubiquitous expression of an ns3-yfp transgene. (*) $P<0.0001$ for $n s 3$ mutant ( $n=6$ groups) compared with control $(n=3$ groups $)$ or $n s 3$ mutant + NS3-YFP (tub) ( $n=6$ groups). Bar: $D, 100 \mu \mathrm{m}$. Data in $C, F$, and $G$ are represented as mean \pm SEM.

\section{Disruption of the ns3 gene impairs larval growth}

In order to analyze the role of NS3 in growth control, we used a fly strain that has a P-element insertion (G0431) (Fig. 1B) in the $n s 3$ gene. We used inverse PCR to map the P-element insertion site to the second exon of ns3, where it is predicted to result in a reading frame shift that removes $88 \%$ of the coding potential of the gene (Supple- mental Fig. S3), thus very likely eliminating the gene's function. We confirmed that the mutant phenotype is in fact due to a dearth of $n s 3$ function with a rescue experiment, described below.

For simplicity, the allele of ns3 containing this P-element insertion (ns $3^{\mathrm{G} 0431}$ ) will be referred to as the $n s 3$ mutant allele from this point forward. The ns3 gene is located on the $\mathrm{X}$ chromosome. Thus, both hemizygous 
mutant males and homozygous mutant females will be referred to as ns3 mutant animals. Except for those shown in Supplemental Figures S4 and S6, A and C, all experiments using adults were performed in males. Experiments with embryos and larvae used mixed populations of males and females.

We generated ns3 mutant animals and examined their development. Mutation of ns3 had no obvious effect on embryonic development, but profoundly retarded larval growth. ns3 mutant animals reached only half the length of their heterozygous counterparts after $5 \mathrm{~d}$ of development (Fig. 1C). Imaginal discs from ns3 homozygotes were reduced in size to a similar degree (Fig. 1D). To evaluate the impact that loss of ns 3 function has on developmental timing, viability, and adult size, we allowed ns3 mutant and control animals raised together under uncrowded conditions to develop to adulthood. ns3 mutant animals exhibited a profound developmental delay, with a median time from egg lay to eclosion of $25.5 \mathrm{~d}$, compared with $13.5 \mathrm{~d}$ for control animals (Fig. 1E, data from males are shown; data from females are similar; Supplemental Fig. S4A). Many ns3 mutant animals died before reaching adulthood; we observed only $30 \%$ of the number of adults that would have been expected based on Mendelian genetics (Fig. 1F, the viability of females was even lower; Supplemental Fig. S4B). Closer examination revealed that most of the "missing" animals died as third instar larvae or as pupae (data not shown).

The ns3 mutant animals that survived to adulthood were properly proportioned and apparently healthy. The mutants are active and do not have a reduced life span, but they are $<60 \%$ of the weight of control animals (Fig. 1G). The defects observed in the ns 3 mutant animals were, in fact, due to loss of NS3 function, because ubiquitous expression of a NS3-YFP fusion protein in ns3 mutant animals completely rescued the developmental delay, reduced viability, and growth defects (Fig. 1E-G). Expression of NS3-YFP in wild-type animals does not result in animals of greater than normal size. These results define a role for $n s 3$ in determination of larval growth rate and adult size.

\section{Cell size and number are reduced in ns3 mutant animals}

To ascertain whether the reduced size of the ns3 mutant animals results from a decrease in cell size, cell number, or both, we measured cell size and number in the wings of ns3 mutant animals. The fly wing is an excellent tissue for this purpose, as each surface of the wing is a monolayer of epithelial cells with a single hair per cell. Mean cell area can be determined by counting the number of wing hairs in a defined area. We determined cell area in this manner by counting the number of wing hairs in $40 \times 40$ - $\mu \mathrm{m}$ squares ( $n=74$ squares for wild-type wings, 99 for $n s 3$ mutant wings) in the area between wing veins vII and vIII. We found that mean cell area was reduced by $16 \%$ in $n s 3$ mutant wings compared with control wings (Fig. 2A). Modeling wing epithelial cells as having a cylindrical shape and a decrease in cell height
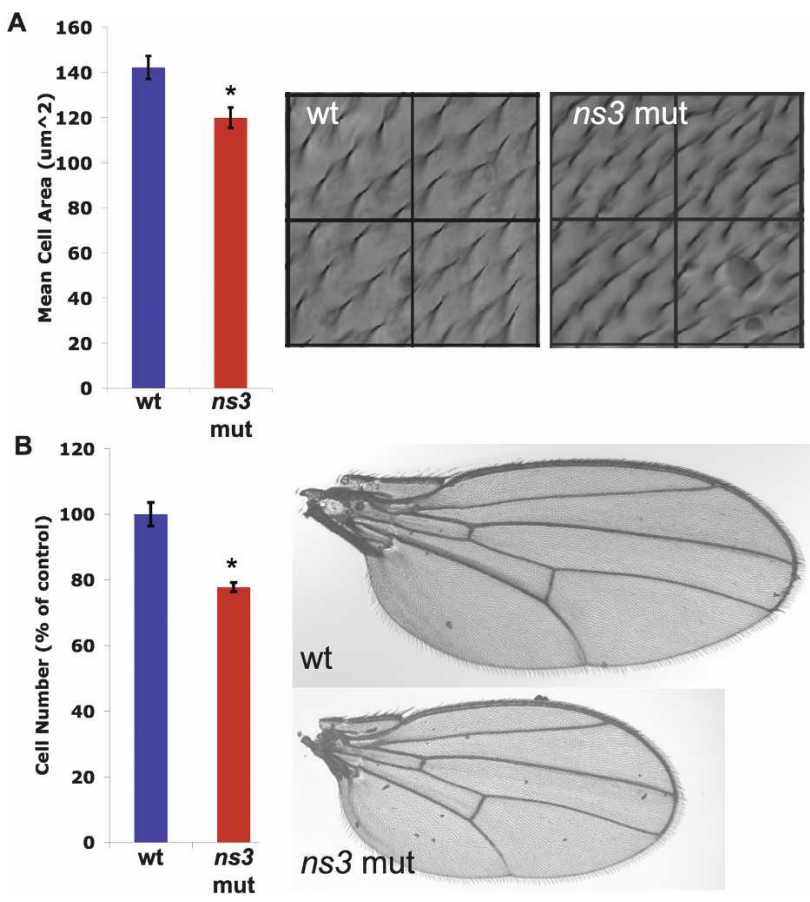

Figure 2. Cell size and number are reduced in $n s 3$ mutant wings. (A) Average cell size was determined in the compartment between veins vII and vIII of wild-type or mutant adult wings from male flies. (Right) Images $(80 \times 80 \mu \mathrm{m})$ from wild-type (wt) and ns3 mutant (ns3 mut) wings. (Left) Quantification of cell area in wild-type and ns3 mutant wings. $(B)$ Average cell number was determined by dividing the total wing area of wild-type and $n s 3$ mutant wings by the calculated cell area from $A$. (Right) Images of wild-type and ns3 mutant wings. (Left) Quantification of cell number in wild-type and ns3 mutant wings. $\left({ }^{*}\right)$ $P<0.0001$ for wild type ( $n=3$ groups) compared with $n s 3 \mathrm{mu}-$ tant $(n=6$ groups). Data are represented as mean \pm SEM.

proportional to the measured decrease in cell radius, we calculated that cell volume would be reduced by $23 \%$ in ns3 mutant wings. We calculated total cell number in the wings by measuring the total wing area and dividing it by the cell area determined above. We found that cell number was reduced by $22 \%$ in the ns3 mutant wings compared with control wings (Fig. 2B). Similar reductions in cell size and number were also observed in the eyes of ns3 mutant animals (Fig. 7, below). Taken together, the $23 \%$ decrease in cell volume and $22 \%$ decrease in cell number would be expected to result in a $\sim 50 \%$ reduction in total tissue volume, sufficient to fully account for the reduced weight of the ns 3 mutant flies.

\section{NS3 acts non-cell-autonomously}

Many regulators of cell growth act cell-autonomously to regulate growth of the cell in which they are expressed. A more limited number have instead been found to function non-cell-autonomously, acting in a subset of cells to coordinate growth throughout the organism /Galloni and Edgar 1999). If NS3 acts at the level of ribosome biogen- 
esis like its yeast ortholog Lsg1p (Kallstrom et al. 2003; Hedges et al. 2005), we would expect it to be a cellautonomous growth regulator.

To address the question of whether NS3 acts cell-autonomously or nonautonomously to control growth, we used two complementary approaches. First, we produced NS3-YFP in subsets of cells in otherwise ns3 mutant animals. Although production of NS3-YFP in all cells using a tubulin-Gal4 driver completely rescued the growth defects of ns3 mutant animals (see Fig. 1), expressing NS3-YFP in several discrete cell populations within ns3 mutants did not result in rescue of cell growth. ns3 mutant animals producing NS3-YFP in larval wing imaginal discs, and adult wings using a 71B-Gal4 driver (Brand and Perrimon 1993) had small wings indistinguishable in size from those of ns3 mutants (Fig. 3A). Similarly, production of NS3-YFP in ns3 mutants in the posterior compartment of larval wing imaginal discs and adult wings using an EngrailedGal4 driver (Tabata et al. 1995) did not increase cell size in the posterior wing (Fig. 3B). In all cases, strong expression of NS3-YFP could clearly be observed in the expected pattern (data not shown). These data indicate that reintroduction of NS3 function into ns3 mutant animals is unable to rescue growth in a cell-autonomous manner.

We next used a loss-of-function approach to further address the question of cell autonomy. We used FLPFRT-mediated mitotic recombination (Golic and Lindquist 1989) to generate ns3 mutant cell clones and wildtype twin-spot cell clones in otherwise ns3 heterozygous animals. The mutant cells are marked by having two copies of GFP, while the wild-type twin-spot cells have no GFP. Surrounding heterozygous cells have a single copy of GFP. If NS3 acts cell-autonomously to control growth, ns3 mutant cell clones would be expected to grow more slowly, and as a result end up smaller than their wild-type twin-spot clones. However, ns3 mutant and wild-type clones were indistinguishable in size (Fig. $3 \mathrm{C}$ ), confirming that the absence of NS3 from patches of wing imaginal disc cells does not change their size. Thus, NS3 function in other cells is evidently adequate for wing imaginal disc cells to be normal in size. NS3 acts non-cell-autonomously.
A

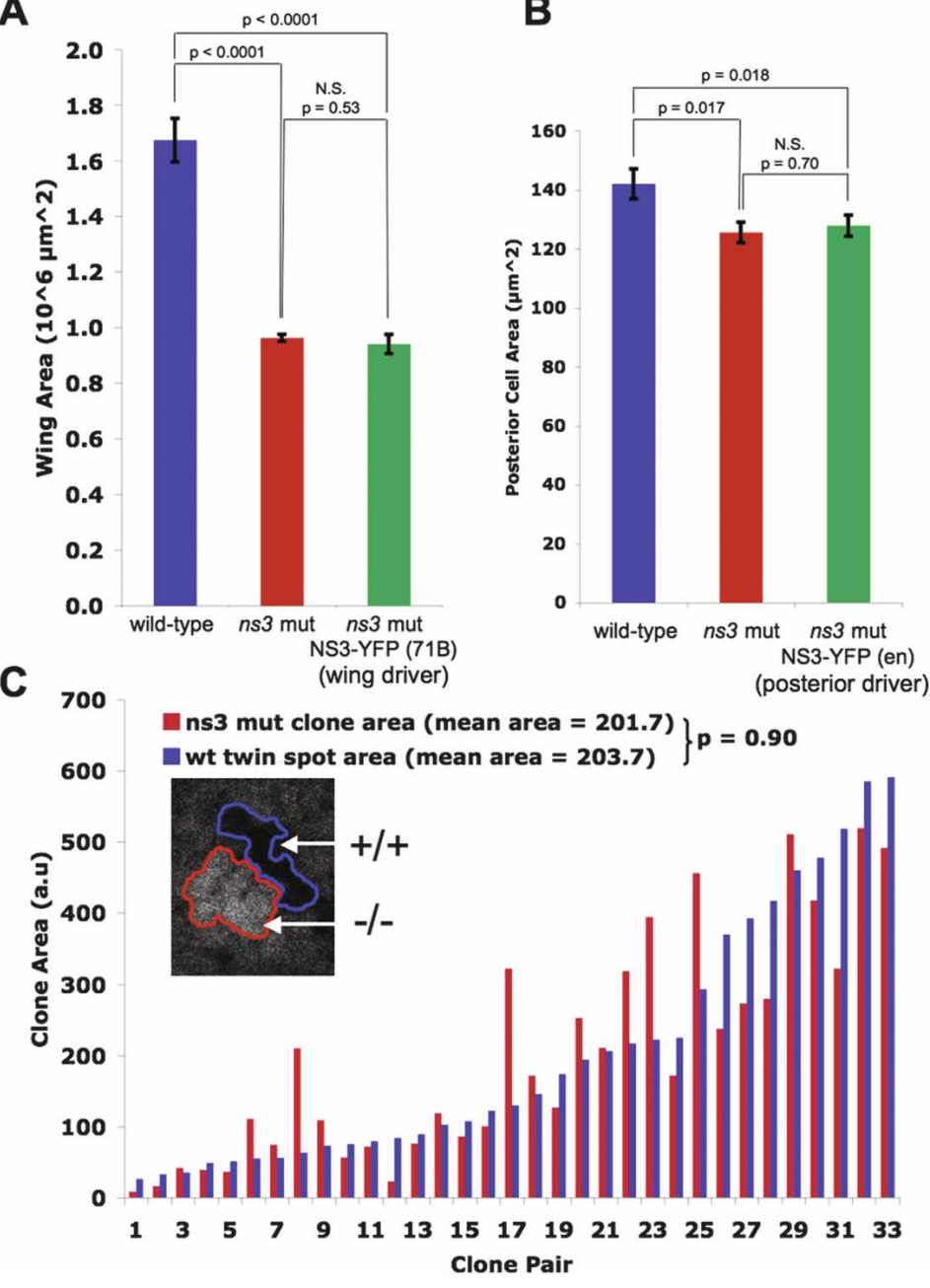

Figure 3. NS3 regulates growth in a non-cell-autonomous manner. (A) Production of NS3-YFP in larval wing imaginal discs and adult wings of otherwise ns3 mutant animals using a 71B-Gal4 driver (71B) did not rescue the reduced wing size of the ns3 mutants. $(B)$ Production of NS3-YFP in the posterior compartment of larval wing imaginal discs and adult wings of ns 3 mutants using an Engrailed-Gal4 driver (en) did not lead to an increase in cell size in the posterior of the wing. (C) FLP-FRT-mediated recombination was used to generate $n s 3^{-/-}$cell clones and wild-type twin-spot clones in otherwise ns3 heterozygous animals. The ns 3 mutant and wild-type clones were indistinguishable in size, indicating that absence of NS3 from patches of wing cells does not change their size. $n s 3^{-/-}$cell clones are marked by two copies of GFP, while wild-type twin-spot clones lack GFP. Surrounding heterozygous tissue has one copy of GFP. Data in $A$ and $B$ are represented as mean \pm SEM. In $A, n=3$ groups for wild type, 4 for ns 3 mutant, and 6 for $n s 3$ mut NS3-YFP (71B). In $B, n=3$ groups for wild type, 6 for $n s 3$ mutant, and 4 for ns3 mut NS3-YFP (71B). 
Kaplan et al.

Figure 4. NS3 acts in serotonergic neurons to control developmental timing. Shown are growth curves of control animals (blue lines), ns 3 mutant animals (harboring, but not expressing, a UAS-NS3-YFP transgene) (red lines), or ns3 mutant animals expressing a UAS-NS3YFP transgene under the control of the indicated tissuespecific Gal4 driver (green lines). The percent of adult animals emerging is represented on the $Y$-axis and the number of days after egg lay (AEL) is represented on the $X$-axis. Only expression of NS3-YFP in all neurons $(C$, ELAV-Gal4) or in serotonergic neurons (E, DDC-Gal4) results in a rescue of the developmental delay exhibited by $n s 3$ mutant animals.
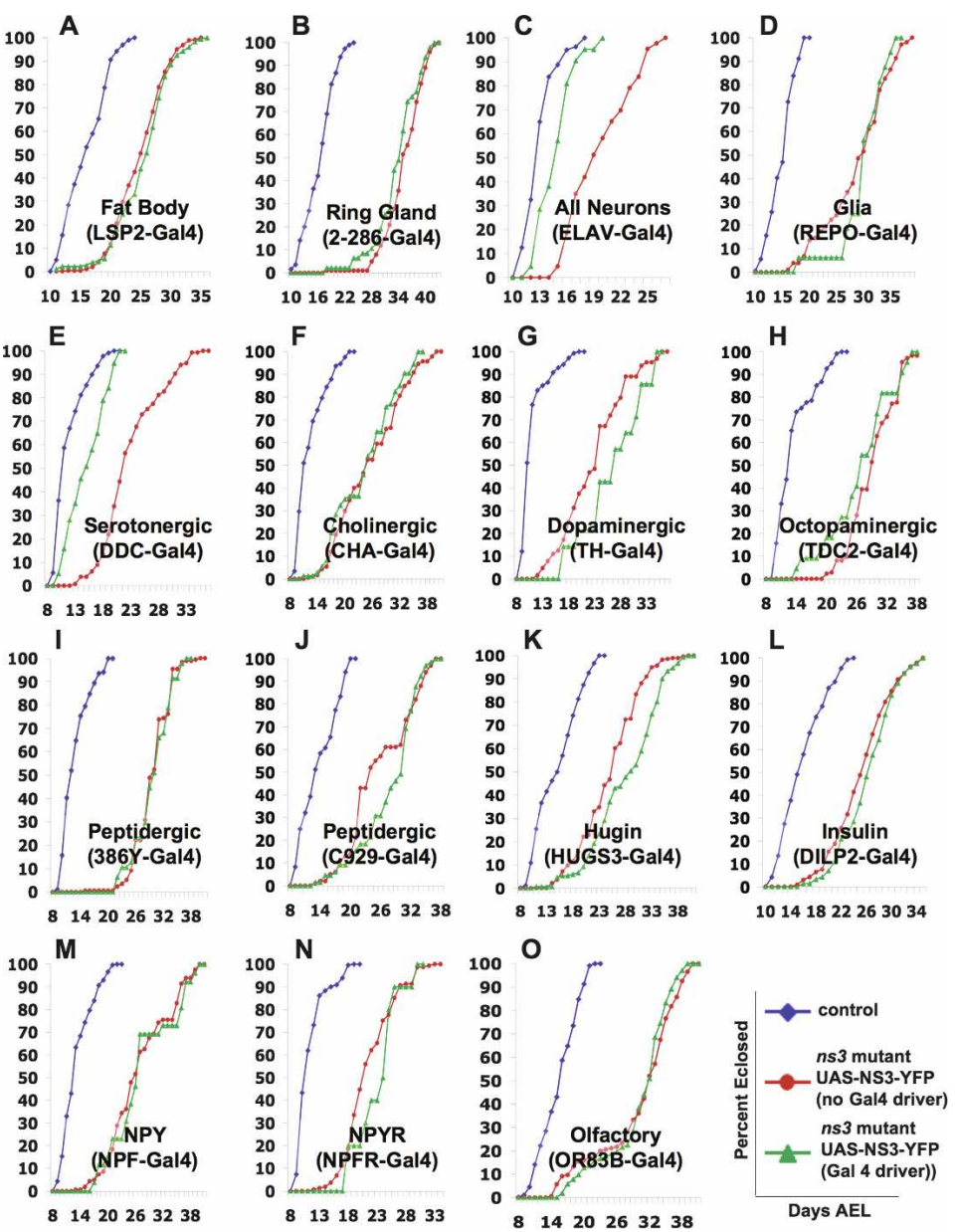

\section{NS3 acts in neurons to control organismal growth}

The nonautonomous action of NS3 in growth control suggests that NS3 may function in a discrete "growth control signaling center" (GCSC) that produces signals that control growth throughout the rest of the organism. The center may also receive, directly or indirectly, sensory and environmental inputs that contribute to growth/no growth decisions. Examination of the localization of ns3 transcript by in situ hybridization revealed that ns 3 mRNA is expressed quite broadly (Supplemental Fig. S5). Because these data did not allow us to pinpoint the location in which NS3 might act to control growth, we instead used a tissue-specific rescue strategy to identify the GCSC. We examined three major growth regulatory centers to determine whether they contain the GCSC in which NS3 acts. The Drosophila fat body is a metabolic organ analogous to the vertebrate liver and adipocytes that coordinates nutrient availability with tissue growth (Colombani et al. 2003). We found that production of NS3-YFP in the fat body of otherwise ns3 mutant animals, using LSP2-Gal4 (Cherbas et al. 2003; Reiling and Hafen 2004), was unable to rescue the developmental delay (Fig. 4A) and growth defects (Fig. 5A) of the mutant animals. Next, we asked whether production of NS3-YFP in the ring gland, an endocrine organ respon- sible for production of growth regulatory hormones including 20-hydroxyecdysone, juvenile hormone, and adipokinetic hormone (Siegmund and Korge 2001; Kim and Rulifson 2004), could rescue the ns3 mutant phenotype. Expression of NS3-YFP in the ring gland using the 2-286Gal4 driver (Timmons et al. 1997; Huang et al. 2005) provided no rescue for the developmental delay (Fig. 4B) and growth defects (Fig. 5B) observed in ns3 mutant animals.

The CNS is involved in many aspects of growth control, from nutrient sensing and appetite to the production of neuroendocrine growth factors (Edgar 2006; Melcher et al. 2007). Thus, we asked whether the nervous system might contain the GCSC. We produced NS3-YFP in neurons of otherwise ns3 mutant animals using ELAV-Gal4, which drives expression in all postmitotic neurons, though not evenly (Sink et al. 2001), and examined the development of these animals. We found that ELAV-driven expression of NS3-YFP resulted in substantial, though not complete, rescue of the developmental delay observed in ns3 mutants (Fig. 4C). Furthermore, ns3 mutant animals expressing NS3-YFP in neurons were significantly larger than their wholly mutant counterparts (Fig. 5C). Importantly, expression of NS3-YFP in glia of ns3 mutants using a REPO-Gal4 driver (Xiong et al. 1994) provided no rescue of the de- 
velopmental delay (Fig. 4D) or size defects (Fig. 5D) observed in these animals, indicating that NS3 functions specifically in neurons to control growth.

\section{NS3 acts specifically in serotonergic neurons} to control body size

To identify which neurons NS3 acts in to control growth, we performed a small-scale rescue screen in which we expressed NS3-YFP individually in a variety of different neuronal subtypes of otherwise ns3 mutant animals and asked whether this limited reintroduction of NS3 function could rescue the developmental delay and growth defects of the mutant animals. No rescue was observed upon production of NS3-YFP in cholinergic neurons (Figs. 4F, 5F; Salvaterra and Kitamoto 2001), dopaminergic neurons (Figs. 4G, 5G; Friggi-Grelin et al. 2003), octopaminergic neurons (Figs. 4H, 5H; Cole et al. 2005), two different subsets of peptidergic neurons (Figs. 4I,J, 5I,J; Taghert et al. 2001), hugin-producing neurons (Figs. 4K, 5K; Melcher and Pankratz 2005), insulin-producing neurons (Figs. 4L, 5L; Rulifson et al. 2002), neuropeptide Y-producing neurons (Figs. 4M, 5M; Wu et al. 2003), neuropeptide $Y$ receptor-expressing neurons (Figs. $4 \mathrm{~N}, 5 \mathrm{~N}$; Wu et al. 2003), or olfactory neurons (Figs. 4O,
5O; $\mathrm{Ng}$ et al. 2002). However, expression of NS3-YFP in serotonergic and dopaminergic neurons using the aromatic L-amino acid decarboxylase promoter (DDC-Gal4) (Li et al. 2000) produced a strong rescue equal to that observed with expression in all neurons (cf. Figs. 4E, 5E and $4 \mathrm{C}, 5 \mathrm{C})$. Because expression in dopaminergic neurons alone gave no rescue (Figs. 4G, 5G), we conclude that NS3 must regulate growth by acting in serotonergic neurons, which make up only 106 of the 250,000 neurons of an adult fly (Valles and White 1988). Consistent with a role for NS3 in serotonergic neurons, we found that superimposed on its widespread expression, ns3 mRNA is somewhat enriched in a pattern resembling that of serotonin immunostaining (Supplemental Fig. S5, cf. C and D).

The rescue experiments described above establish that expression of NS3 in serotonergic neurons of ns3 mutant animals is sufficient to rescue their growth defects. We next asked whether NS3 function in serotonergic neurons is necessary for normal growth. We expressed an shRNA (Dietzl et al. 2007) targeting ns3 under control of the DDC-Gal4 promoter to reduce ns3 levels in serotonergic neurons. We found that $n s 3$ knockdown in these neurons resulted in a developmental delay (Supplemental Fig. S5A) and reduced adult size (Supplemental Fig. $\mathrm{S} 5 \mathrm{~B})$, phenotypes reminiscent of (although milder than)
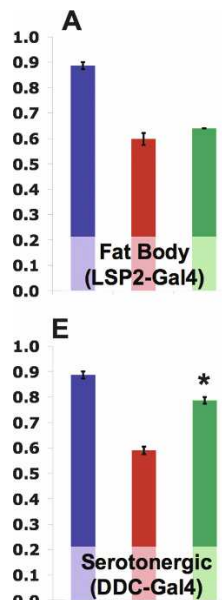

0.0

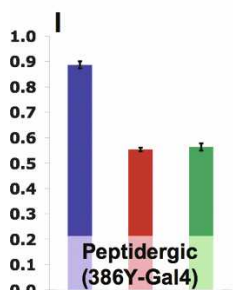

0.0

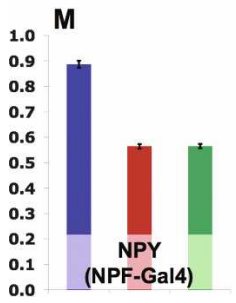

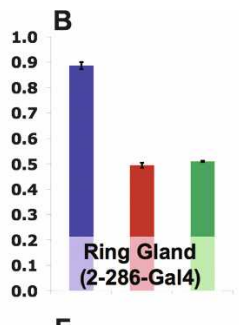

$F$

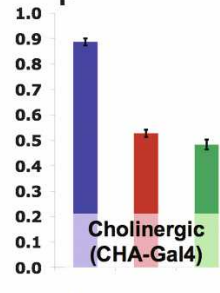

J

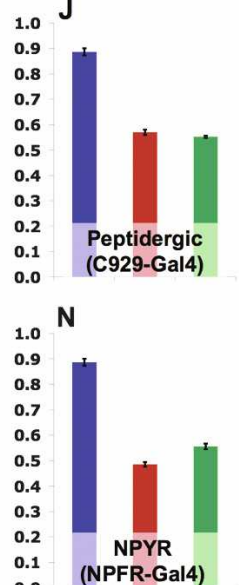

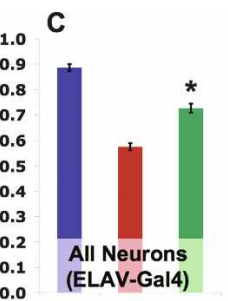

G
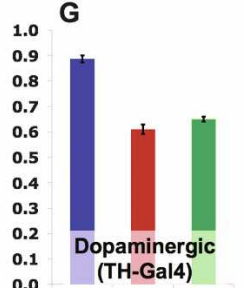

K

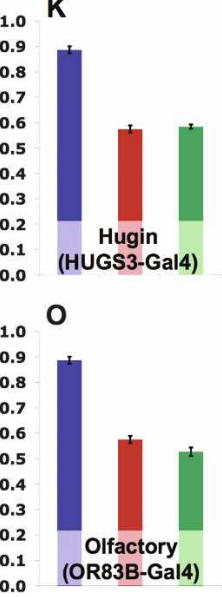

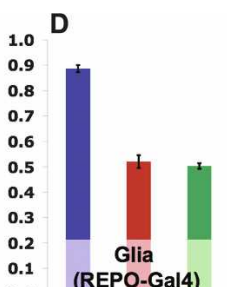
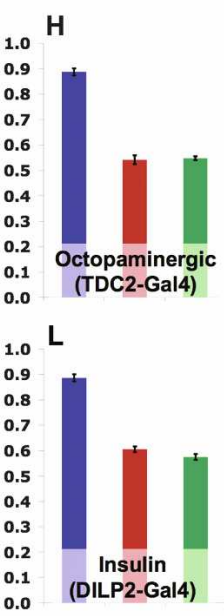

0.0

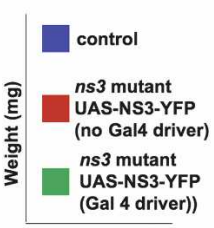

Figure 5. NS3 acts in serotonergic neurons to control organismal growth. Average weights of control animals (blue bars), ns3 mutant animals (harboring, but not expressing, a UAS-NS3-YFP transgene) (red bars), or ns3 mutant animals expressing a UAS-NS3-YFP transgene under the control of the indicated tissue-specific Gal4 driver (green bars) are shown. Only expression of NS3YFP in all neurons ( $C$, ELAV-Gal4) or in serotonergic neurons $(E, \mathrm{DDC}-\mathrm{Gal})$ results in rescue of the reduced weight exhibited by ns3 mutant animals. Data are represented as mean \pm SEM. $\left({ }^{\star}\right) P$-values: $(C)$ ns3 mut $(n=4$ groups) versus $n s 3$ mut + NS3-YFP (ELAV) ( $n=5$ groups), $P=0.0007$. (E) ns3 mut $(n=3$ groups $)$ versus $n s 3$ mut + NS3-YFP (DDC) $(n=3$ groups $), P=0.0002$. 
those observed in the ns3 mutant animals. The milder phenotype observed with the shRNA compared with the ns3 mutant phenotype is likely due to a hypomorphic effect resulting from incomplete mRNA or protein knockdown, a phenomenon commonly observed with shRNA expression in Drosophila (Dietzl et al. 2007). We conclude that NS3 function in serotonergic neurons is both necessary and sufficient for its role in growth control.

\section{Serotonin levels are elevated in ns3 mutants}

Serotonin (5-hydroxytryptamine, or 5-HT) is a monoamine neurotransmitter synthesized from the amino acid tryptophan. In both mammals and flies, serotonergic neurons project widely and regulate a variety of behaviors, including learning and memory, circadian rhythms, sleep, feeding, aggression, and responses to psychoactive drugs (Monastirioti 1999; Sanders-Bush and Mayer 2001). In humans, pharmacological modulation of serotonergic function is used in the treatment of obesity and many psychiatric disorders (Schwartz et al. 2000; Sanders-Bush and Mayer 2001). In Drosophila, perturbation of serotonergic function slows larval growth (Valles and White 1986). The requirement for NS3 function in serotonergic neurons led us to ask whether serotonin levels are perturbed in the brains of ns3 mutant animals. We used an ELISA to measure serotonin content in heads from wild-type or ns 3 mutant flies and found that levels of serotonin per body weight were increased by $\sim 50 \%$ in ns3 mutants (Fig. 6A).

To examine the influence of increased serotonin on growth, we increased serotonin levels in wild-type or ns3 mutant animals by feeding them the immediate precursor of serotonin, 5-hydroxy-L-tryptophan (5-HTP), and measured the duration of development from embryos to pupae. We found that 5 -HTP treatment at the highest doses used (1.0 and $2.0 \mathrm{mg} / \mathrm{mL}$ ) resulted in a mild developmental delay in wild-type animals (Supplemental Fig. S7A). ns3 mutant animals were considerably more sensitive to increasing serotonin levels, with a strong dosedependent developmental delay in response to 5-HTP treatment (Supplemental Fig. S7B). These data are consistent with elevated serotonin having a growth inhibitory effect, and with the elevated levels of serotonin observed in the ns3 mutants being responsible for the growth defects observed in these animals.

\section{Projections from serotonergic neurons and ipcs are closely apposed}

Disruption of insulin production in flies results in a phenotype very similar to that observed in ns3 mutants, including retarded larval growth, delayed development, and reduced adult size (Ikeya et al. 2002; Rulifson et al. 2002). A number of recent studies have suggested that connections exist between serotonin and insulin signaling, but the precise nature of these connections is unclear. Sze et al. (2000) demonstrated that in Caenorhab- ditis elegans, ectopic activation of insulin-like signaling could suppress phenotypes associated with loss of serotonin, suggesting that serotonin may be acting as an upstream regulator of insulin-like signaling. This finding is consistent with work in rats showing that increasing serotonin levels can lead to an increase in insulin synthesis and/or release (Peschke et al. 1997; Orosco et al. 2000). Because NS3 acts in serotonergic neurons but loss of NS3 phenocopies IPC ablation, and direct expression of NS3 in IPCs does not rescue the ns3 mutant phenotype (Figs. 4L, 5L), we investigated the anatomical arrangement of serotonergic neurons and IPCs in the Drosophila brain. The goal was to determine the potential for communication between these two neuronal signaling systems. IPCs were detected by producing GFP under the control of a dilp2 promoter (Rulifson et al. 2002), and serotonergic neurons were detected by immunostaining for serotonin (Neckameyer et al. 2007).

We found that projections from serotonergic neurons and IPCs are closely apposed in the brain lobes (Fig. 6B,C) and in the ventral ganglion (Fig. 6B,D). In each brain lobe, a dense neuropil containing many serotonergic processes is present in close proximity to the IPCs /arrowheads in Fig. 6C). Processes from the IPCs and serotonergic neurons also exhibit extensive regions of apposition in the commissure between the brain lobes (arrow in Fig. 6C) and along the length of the ventral ganglion (arrowheads in Fig. 6D). Serotonin is not typically released from synaptic terminal boutons, but rather from varicosities throughout the neuropil, allowing for modulation of numerous target synapses over a relatively broad area (Sykes and Condron 2005). The IPCs and their processes are thus favorably positioned to receive input from the serotonergic neurons.

\section{Insulin levels are elevated in ns3 mutants}

The elevation of serotonin levels in ns 3 mutants and the close apposition of the serotonergic and IPC processes prompted us to examine whether insulin pathway function is altered in $n s 3$ mutant animals. We examined the amount of insulin in the brains of wild-type and ns3 mutant larvae by immunostaining whole-mount brains using an antibody to DILP2, imaging the brains by confocal microscopy using identical laser power and scan settings, and then quantifying fluorescence intensity in the IPCs. We found that DILP2 levels were more than sevenfold higher in the ns3 mutants compared with wild-type animals (Fig. 7A,B,G), a surprising finding given that the ns3 mutant phenotype resembles that of animals lacking insulin. In addition to having increased levels of DILP2 in the IPCs, ns 3 mutant animals also had high levels of DILP2 in the processes emanating from the IPCs (Fig. 7, cf. D and E). To further examine the functional linkage between NS3 activity in serotonergic neurons and IPC function, we examined DILP2 levels in the IPCs of "rescued" ns3 mutant animals expressing NS3YFP in serotonergic neurons under control of a DDCGal4 driver. We found that DILP2 levels in these animals 

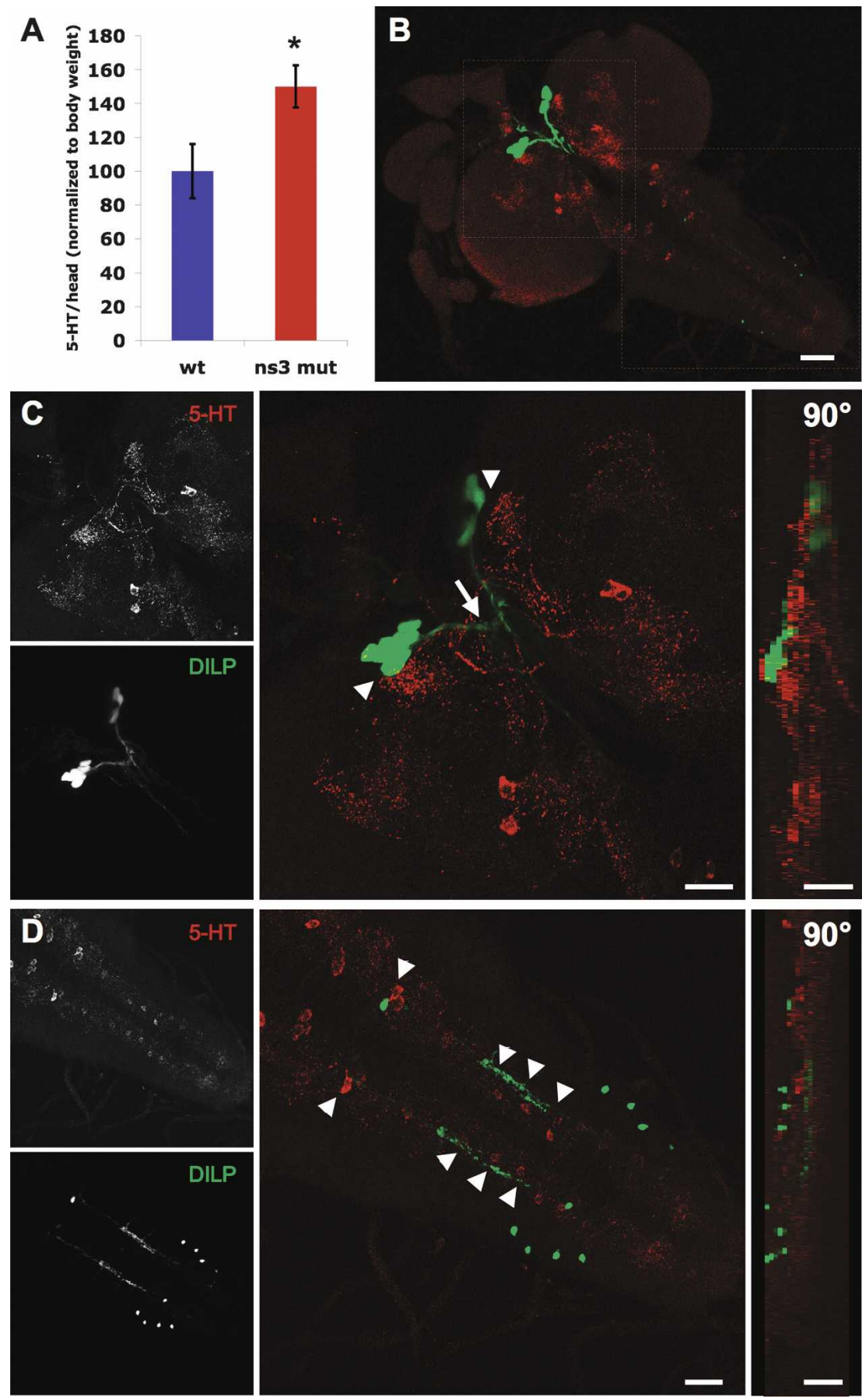

Figure 6. Projections from serotonergic neurons and IPCs are closely apposed. (A) Serotonin levels are elevated in $n s 3$ mutants. Levels of serotonin $(5-\mathrm{HT})$ were measured by ELISA and are represented as mean \pm SEM per head, normalized to body weight. $\left({ }^{\star}\right) P<0.05(n=4$ groups each). (B) Low-magnification 3D projection of a confocal Z-stack of a wholemount wild-type Drosophila brain from a wandering third instar larva expressing GFP in the IPCs under control of a dilp2 promoter (green) and immunostained for 5-HT (red). $(C, D)$ Higher-magnification images of brain lobes $(C)$ and ventral ganglion $(D)$, acquired as in $A$. Arrowheads in $C$ denote regions where the neuropil containing serotonergic processes is in close proximity to the IPCs. Arrow in $C$ denotes apposition of processes from the IPCs and serotonergic neurons in the commissure between the brain lobes. Arrowheads in $D$ denote regions of apposition between serotonergic and IPC processes along the length of the ventral ganglion. Individual channels are shown in grayscale images at left. Ninety-degree rotation about the $Y$-axis of the Z-stacks are shown at right. Note that the serotonergic and IPC projections are in the same plane. Bars: $B, 40 \mu \mathrm{m} ; C, D, 20 \mu \mathrm{m}$. were reduced significantly compared with ns 3 mutants, although they were still somewhat higher than in wildtype animals (Fig. 7C,F,G). This result shows that high levels of DILP2 do not occur in a ns3 mutant animal where nearly all cells lack the rescuing NS3-YFP, indicating that DILP2 accumulation is dependent only on NS3 function in serotonergic neurons and not on signals from the periphery. To determine whether the increased DILP2 protein level in ns3 mutant animals was caused by increased transcription of the dilp2 gene, we used quantitative real-time PCR to measure dilp2 transcript levels. We found that the level of dilp2 transcript in ns3 mutants was indistinguishable from that of wildtype animals (ns3 mutants' dilp2 transcript level was $1.10 \pm 0.13$-fold of wild type, $P=0.49[n=3])$, indicating that the increased DILP2 protein in ns3 mutants is not caused by increased expression of the dilp2 gene.

The data presented above indicate that signaling from serotonergic neurons to IPCs is important for normal growth. To further demonstrate that this communication is important, and that the serotonergic neurons are not themselves controlling growth through production 
Kaplan et al.

of DILP2, we expressed shRNA targeting dilp2 in serotonergic and dopaminergic neurons. We did not observe any growth defects in these animals (Supplemental Fig. S6C,D), consistent with serotonergic neurons controlling growth through regulation of insulin secretion from the IPCs.

Figure 7. NS3 acts in serotonergic neurons as an upstream regulator of insulin signaling. $(A-G)$ DILP2 levels are elevated in ns3 mutant brains. Brains from third instar larvae were immunostained using an antibody to DILP2 and imaged by confocal microscopy using identical laser power and scan settings. High-magnification images are shown in $A-C$, and lower-magnification images are shown in $D-F$, and in these images, DILP2 is shown in green and DNA counterstain is shown in blue. Fluorescence intensity in the IPCs is quantified in $G .\left(^{*}\right)$ $P<0.0001$ for $n s 3$ mutant $(n=35)$ versus wild type $(n=8)$ or for ns3 mutant versus ns3 mutant + NS3YFP (DDC) $(n=30)$. $(H)$ Insulin pathway activation is impaired and small ribosomal subunit protein levels are reduced in $n s 3$ mutant larvae. Immunoblot for indicated proteins from equal amounts of ns3 heterozygous (ns3 het) or mutant (ns3 mut) larval extract. Data are from one experiment that is representative of three independent experiments. (I) Akt activation and ribosomal protein S6 (RPS6) levels are not altered in ns 3 mutant cell clones, but Akt activation is reduced in $\mathrm{TOR}^{\Delta \mathrm{P}}$ mutant clones. For $n s 3$ mutant clones, wing imaginal disc tissue in which mitotic clones have been generated is marked as mutant by two copies of GFP (green in merge) or wild type by lack of GFP. Surrounding heterozygous tissue has one copy of GFP. For $\mathrm{TOR}^{\Delta \mathrm{P}}$ mutant clones, wing imaginal disc tissue in which mitotic clones have been generated is marked as mutant by lack of GFP or wild type by two copies of GFP (green in merge). Surrounding heterozygous tissue has one copy of GFP. Tissue was immunostained for phospho-S505-Akt1 (top and bottom panels, red in merge) or RPS6 (middle panel, red in merge). $(J-L)$ Expression of constitutively-active Akt1 T342D, S505D (Akt1) in the eye using a GMR-promoter fusion rescues the reduction in eye size $(J)$, ommatidia size $(K)$, and ommatidia number $(L)$ observed in ns3 mutant flies. Images in $J$ show scanning electron micrographs of whole eyes (eye size quantified at right). Images in $K$ show scanning electron micrographs of ommatidia (ommatidia size quantified at right and ommatidia number is quantified in $L) .\left({ }^{\star}\right) P$-values: $(J)$ wild type versus $n s 3$ mut, $P=0.001$; $n s 3$ mut versus wild type + Akt1, $P<0.0001 ; n s 3$ mut versus ns3 mut + Akt1 $P<0.0001$; wild type + Akt1 versus ns3 mut + Akt $1, P=0.77$. (K) Wild type versus $n s 3$ mut, $P=0.0023 ;$ ns3 mut versus wild type + Akt 1 , $P=0.0009 ; n s 3$ mut versus ns3 mut + Akt1, $P=0.0018$; wild type + Akt 1 versus ns 3 mut + Akt 1 , $P=0.43$. $(L)$ Wild type versus $n s 3$ mut, $P=0.0048$; ns3 mut versus wild type + Akt1, $P=0.0034$; ns3 mut versus ns3 mut + Akt1, $P=0.011$; wild type + Akt1 versus ns3 mut + Akt $1 P=0.55$. Bars: $A-F, 25 \mu \mathrm{m} ; J, 100 \mu \mathrm{m} ; K, 15 \mu \mathrm{m}$. Data are represented as mean \pm SEM. $n=3$ per condition.

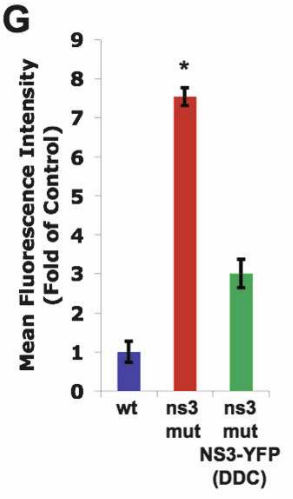

Peripheral insulin pathway activation is reduced in ns3 mutants

We hypothesized that the seemingly paradoxical increase in levels of DILP2 in ns 3 mutant animals could be explained by a defect in the release of DILP2 into the
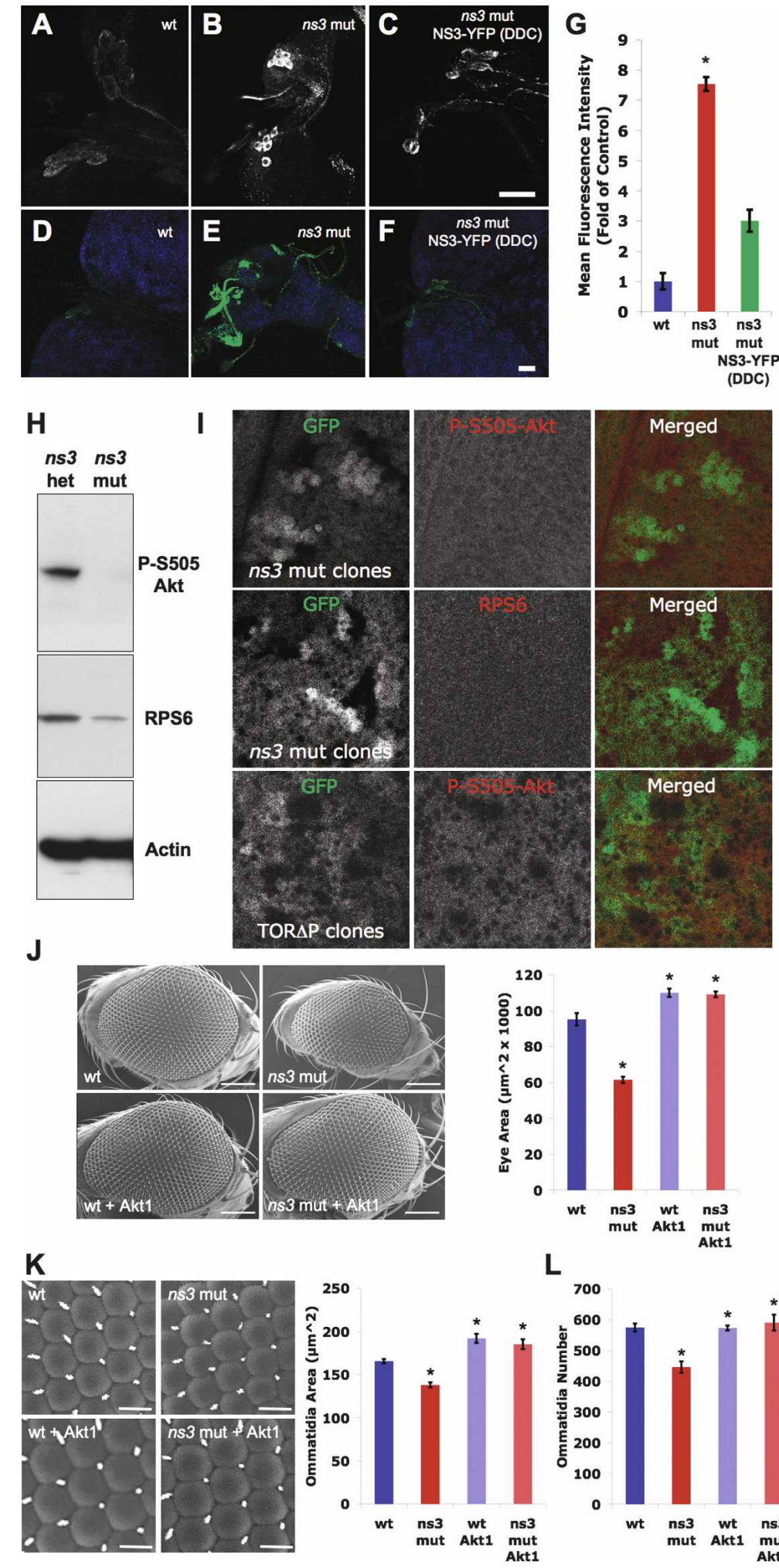

$\mathbf{L}$

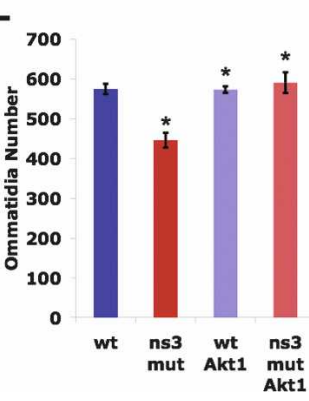


circulation. No assay sensitive enough to detect levels of DILP2 circulating in the hemolymph is currently available, so we used two alternate methods to measure peripheral insulin pathway activation. A central effector of the insulin signaling pathway is the kinase Akt1, which becomes phosphorylated and active in response to insulin. We measured the level of Akt1 activation in larval extracts using an antibody that recognizes only the active, phosphorylated (S505-P) form of the protein. Akt1 activation was impaired in ns3 mutant larvae (Fig. $7 \mathrm{H}$ ).

Active Akt1 influences a number of downstream effectors to stimulate ribosome biogenesis. To determine whether the impaired insulin pathway activation observed in the ns3 mutant larvae led to a defect in ribosome biogenesis, we measured the level of small ribosomal subunits in the larval extracts. The antibody used detects the ribosome protein RPS6, a component of small ribosome subunits that is present at a single copy per ribosome and is thus a faithful marker of total small ribosome subunit number (Wimberly et al. 2000). Small ribosome subunit levels were reduced in ns 3 mutant larvae (Fig. $7 \mathrm{H})$. These data indicate that despite having elevated levels of neuronal insulin, ns3 mutants have reduced peripheral insulin pathway activation, consistent with a defect in insulin release into the circulation.

\section{NS3-mediated regulation of insulin pathway} activation is non-cell-autonomous

NS3 regulates levels of Akt1 activation and small ribosomal subunit abundance in the whole animal (Fig. 7H). We asked whether both types of regulation occur in a cell-autonomous manner. We examined Aktl activation and small ribosomal subunit levels by immunostaining wing imaginal discs from the animals bearing the type of ns3 mutant clones that are described in Figure 3. In contrast to the reduction in active Akt1 and ribosome protein levels observed in extracts of ns3 mutant animals, Akt 1 activation and small ribosomal subunit abundance were indistinguishable in ns3 mutant cell clones as compared with their wild-type twin-spot clones (Fig. 7I, top and middle panels). As a control, we examined Akt1 activation in clones mutant for the cell-autonomous growth regulator TOR (Zhang et al. 2000). As expected in cells mutant for an upstream activator of Akt1 (Sarbassov et al. 2005), Akt1 activation was reduced in $\mathrm{TOR}^{\Delta \mathrm{P}}$ mutant clones (Fig. 7I, bottom panels). These data indicate that NS3 regulates Akt1 activation and small ribosomal subunit levels in a non-cell-autonomous manner.

Activation of insulin signaling in eye cells rescues the ns3 mutant phenotype

To confirm that the reduction in peripheral insulin pathway activation is responsible for the growth defects in ns3 mutants, we asked whether activation of the insulin signaling pathway could rescue the defects in cell growth that is characteristic of ns 3 mutants. Eye cells are small and less numerous in ns3 mutants. We produced constitutively active (T342D, S505D) Akt1 in the eyes of ns3 mutant flies using a GMR driver. We found that $\mathrm{Akt1}^{\mathrm{T} 342 \mathrm{D}, \mathrm{S505D}}$ rescued the defects in total organ size (Fig. 7J), cell size (Fig. 7K), and cell number (Fig. 7L) in eyes of ns3 mutant animals. In fact, organ size, cell size, and cell number in ns3 mutant animals expressing Akt1 ${ }^{\mathrm{T} 342 \mathrm{D}}$, 5505D were as great as in wild-type animals expressing $\mathrm{Akt1}^{\mathrm{T} 342 \mathrm{D}, \text { S505D }}$ (Fig. 7J-L). Organ and cell size were actually greater than in wild-type animals (Fig. $7 \mathrm{~J}, \mathrm{~K})$. Production of constitutively active Akt1 has previously been shown to result in increased cell and organ size (Verdu et al. 1999). These results show that within an organ the function of NS3 in growth control can be fully bypassed by ectopic activation of insulin signaling. Considered in light of our finding that insulin builds up in the brains of $n s 3$ mutant animals while peripheral insulin pathway activation is reduced, these data suggest that the defective insulin pathway activation observed in ns3 mutant animals is the primary cause of their growth defects.

\section{Discussion}

Molecular regulators that operate within the CNS to integrate environmental, nutritional, and physiological information to direct proper growth responses remain for the most part mysterious. We provide evidence here that NS3 is one such molecular regulator.

\section{Nucleostemin 3 acts in the CNS to regulate insulin signaling}

NS3 is part of a large family of YlqF-Related GTPase (YRG) proteins that are evolutionarily conserved in organisms as diverse as bacteria and mammals (Reynaud et al. 2005). Some nucleostemin proteins are located in nucleoli, and a yeast member of the family, Lsglp, has been implicated in ribosome assembly. ns3 mutant animals grow slowly and reach an adult weight only $\sim 60 \%$ that of controls. Based on the role of Lsg1p, we suspected that NS3 would contribute to protein synthesis and thus cell growth. That view predicts a cell-autonomous function of NS3 within cells whose size and number are decreased in ns3 mutants, just as Minute mutations that cause lowered ribosome protein production reduce Drosophila body size (Lambertsson 1998).

Contrary to the cell-autonomous protein synthesis hypothesis, NS3 was found to play its major role in the nervous system. Clones of $n s 3$ mutant cells in peripheral tissues such as eye and wing were normal in size, indicating that NS3 is not required for the growth of most cells, and suggesting instead that NS3 is important for signaling events that influence cell growth and division. To identify where in the animal NS3 acts to control growth, we introduced potentially rescuing $n s 3$ activity into a large variety of cell types and found that rescue occurs only when the protein is expressed in the nervous system. From these findings we also concluded that NS3 is unlikely to be secreted, since in that case ns3 ex- 
pressed in tissues such as muscle, epidermis, or fat body would be expected to provide a rescuing effect.

Our data suggest that NS3 functions in serotonergic neurons as part of the central neural regulation of growth. This conclusion is supported by experiments demonstrating that $(1)$ in the absence of NS3, serotonin and insulin levels in the brain are elevated, while peripheral insulin pathway activation is reduced, and growth is severely disrupted. (2) Reintroduction of NS3 into serotonergic neurons of ns3 mutant animals relieves the buildup of insulin in IPCs and rescues the growth defects in these animals. Thus, a signal from serotonergic neurons controls insulin levels in IPCs. (3) Activation of insulin signaling in target tissues can bypass the growth defects in ns3 mutants. Thus, the action of NS3 in serotonergic neurons lies upstream of insulin signaling in the growth control hierarchy.

\section{A new function for a nucleostemin-family protein}

Human NUCLEOSTEMIN was cloned in a screen for genes that are highly expressed in stem cells and that are repressed upon stem cell differentiation (Tsai and McKay 2002). NUCLEOSTEMIN is abundant in many undifferentiated cell types, is scarce in most differentiated cells, and is important for maintaining stem cells and cancer cells in a proliferative state (Tsai and McKay 2002). Another member of this family, Lsglp, is the yeast protein most closely related to Drosophila NS3. Lsg1p functions in ribosome biogenesis; a role that is essential for growth of yeast cells (Kallstrom et al. 2003; Hedges et al. 2005). Here we show that Drosophila NS3 is a non-cell-autonomous regulator of growth that functions in serotonergic neurons to control body size. How could these three closely related proteins perform such different functions?

One difference may be subcellular protein localization. In eukaryotes, members of the YRG protein family are found in the cytoplasm, mitochondria, nucleus, and nucleolus (Reynaud et al. 2005). NUCLEOSTEMIN is a nucleolar protein (Tsai and McKay 2002), while Lsg1p has a somewhat punctate, cytoplasmic localization (Kallstrom et al. 2003). We found that NS1 and NS2 are concentrated in the nucleolus, while NS3 is primarily located in the cytoplasm, in well-defined puncta (Supplemental Fig. S1). Thus, the localization of Drosophila NS3 is more similar to its yeast ortholog Lsg1p than to human NUCLEOSTEMIN or to the most closely related Drosophila orthologs, NS1 and NS2.

How, then, can the striking difference in function between Lsg1p and NS3 be explained? Although NS3 and Lsglp regulate growth in very different ways, they may be acting similarly at a molecular level. In addition to a function in ribosome biogenesis, Lsg1p may play a role in translation (Kallstrom et al. 2003). It is possible that NS3 also regulates translation, but to a different end. Lsg1p and NS3 share a conserved RNA-binding domain (Supplemental Figs. S1, S3). Using this domain, NS3 could, for example, regulate the translation of a factor involved in serotonin metabolism or release. Identifying proteins and/or RNAs bound to NS3 in serotonergic neurons may be a means of addressing this question.

\section{NS3 mutation reveals central growth control machinery}

Serotonin modulates the development and function of a number of different neuronal signaling systems (Monastirioti 1999; Sanders-Bush and Mayer 2001). Most serotonergic cells are interneurons acting entirely within the CNS (Valles and White 1988), where they serve an important processing role-integrating informational inputs and converting them into instructive outputs. We propose that signals from serotonergic neurons modulate insulin secretion, control growth, and thus coordinate the formation of proportional sizes of tissues and organs.

Reintroduction of NS3 into only 106 serotonergic neurons substantially rescued the growth defects seen in $n s 3$ mutant animals. Complete rescue might be achieved through additional expression in certain other, as yet unknown cells, or might depend on precise times or amounts of production in the serotonergic neurons. Even relatively imprecisely controlled NS3 production in serotonergic neurons provides as much rescue as expression in the entire nervous system and restores close-tonormal body size, so we believe we found the main location of NS3 action with respect to body size control. The DDC-Gal4 driver that we used to drive expression in serotonergic neurons also drives expression in dopaminergic neurons ( $\mathrm{Li}$ et al. 2000). Because expression in dopaminergic neurons alone gave no rescue, we concluded that NS3 acts specifically in serotonergic neurons to control growth. However, the possibility exists that NS3 activity is required simultaneously in both serotonergic and dopaminergic neurons. Once a Gal4 transgene that drives expression only in serotonergic neurons is available, this question can be addressed.

Projections from serotonergic neurons are closely apposed with those of insulin-producing neurons (IPCs), so IPCs are favorably positioned to receive direct input from serotonergic neurons and indeed to send signals back. In accordance with control of insulin secretion by serotonergic neurons, we observed that the brains of ns3 mutants contain higher than normal serotonin and insulin, while peripheral insulin pathway activation was reduced. Local, peripheral activation of Akt, a kinase that functions as a key transducer of the insulin signaling pathway, rescued the growth defects of ns3 mutants. These data show that the ability of insulin pathway transduction to drive growth is preserved in the mutants. NS3 acts within the CNS to control growth signaling via the insulin pathway, and thus exerts global control over cell size and number.

While we suggest that the buildup of insulin observed in $n s 3$ mutants is due to a defect in insulin secretion, the tools necessary to address this question directly do not yet exist. Once an assay is developed that is sensitive enough to detect circulating levels of Drosophila insulins, it will be possible to measure changes in insulin levels in response to acute manipulation of serotonin 
function or genetic perturbations that affect the serotonin or insulin signaling pathways, such as ns3 mutation. Methods for control of protein production in subsets of serotonergic neurons would allow the identification of the specific serotonergic neurons that contribute to insulin regulation. At that time it will be useful to do electron microscope reconstruction experiments to trace the connections of the relevant neurons with target neurons.

Our results are consistent with either positive or negative regulation of insulin secretion by serotonin. If serotonin negatively regulates insulin secretion, the elevated serotonin levels in $n s 3$ mutants would be expected to inhibit insulin secretion, which could explain the high levels of insulin in the IPCs and the defect in peripheral insulin signaling. Alternatively, if serotonin is a positive regulator of insulin secretion, our results could be explained by a defect in serotonin release in the $n s 3 \mathrm{mu}-$ tants. In such a scenario, the defect in serotonin release would deprive IPCs of stimulation. The consequent failure to secrete insulin would block peripheral insulin pathway activation.

\section{Connecting serotonergic neuron function to insulin signaling}

Work with both C. elegans and mammals supports the existence of a connection between serotonin and insulin signaling, although the nature of this connection remains unclear. In C. elegans, mutation of the insulin receptor gene $d a f-2$ results in increased reproductive longevity that is dependent on DAF-16, a forkhead transcription factor that carries out a program of gene expression that is inhibited in response to insulin signaling (Kenyon et al. 1993). Worms defective in serotonin synthesis exhibit increased reproductive longevity, an effect that can be reversed by mutation of daf-16 (Sze et al. 2000), which mimics aspects of insulin pathway activation. Studies in mammals have produced contradictory reports about the effect of serotonin on insulin secretion. Studies of rabbit pancreas slices and rat islets of Langerhans found that exposure to serotonin resulted in increased insulin secretion (Telib et al. 1968; Gagliardino et al. 1971; Peschke et al. 1997). On the other hand, work using golden hamster pancreases found that serotonin inhibited insulin secretion (Feldman and Lebovitz 1970).

In mammals, insulin acts via the insulin receptor to regulate blood glucose, while the related insulin-like growth factors (IGFs) act through IGF receptors to stimulate cell growth. However, in Drosophila, regulation of both hemolymph glucose and cell growth are controlled by the DILPs, acting via a single insulin receptor (Baker and Thummel 2007). Interestingly, serotonin increases IGF-I release from human granulosa cells (Schaeffer and Sirotkin 1997). Thus, despite the separation of glucose regulation from growth control in mammals, serotonin influences both. The serotonin-insulin connection may be an example of evolutionarily conserved physiology.

\section{Materials and methods}

Fly lines used

The following fly lines were used: Canton-S, $\mathrm{w}^{1118}$, yw, P[lacW]1(1)G0431 ${ }^{\text {G0431 }} \mathrm{w}^{67} \mathrm{c}^{23} / \mathrm{FM} 7 \mathrm{c}, \mathrm{w}^{1118}$; PBac[PB]CG9320 ${ }^{\text {c06563' }}$ FM7i，P[ActGFP]JMR3/C(1)DX, $\mathrm{y}^{1} \mathrm{f}^{1}$, GMR-dAkt1 ${ }^{\mathrm{T} 342 \mathrm{D}-\mathrm{S505D}}$ ', $\mathrm{P}\left[\right.$ neoFRT]18A; ry ${ }^{506}, \mathrm{pr}^{1} \mathrm{pwn}^{1} \mathrm{P}[\mathrm{hsFLP}] 38 / \mathrm{CyO} ; \mathrm{Ki}^{1} \mathrm{kar}^{1} \mathrm{ry}^{506}{ }^{\prime}$ $\mathrm{w}^{1118} \mathrm{P}\left[\right.$ Ubi-GFP(S65T)nls]X P[neoFRT]18A, $\mathrm{y}^{1} \quad \mathrm{w}^{\star} ; \quad$ Tor $^{\Delta \mathrm{P}}$ $\mathrm{P}[$ neoFRT]40A/CyO, Tubulin-Gal4, 71B-Gal4, Engrailed-Gal4, LSP2-Gal4, 2-286-Gal4, ELAV-Gal4, REPO-Gal4, w; P[DDCGal4], w; CHA-Gal4, w; P[ple(TH)-Gal4], w; P[TDC2-Gal4], w; P[386Y-Gal4], C929-Gal4, HUGS3-Gal4, DILP2-Gal4, DILP2Gal4; UAS-GFP, yw ; P[NPF-Gal4], yw; P[NPRF-Gal4], OR83BGal4, UAS-ns3-shRNA, UAS-dilp2-shRNA.

\section{Generation of ns3 mutant animals}

$\mathrm{P}\left[\right.$ lacW]1(1)G0431 ${ }^{\mathrm{G} 0431} \mathrm{w}^{67} \mathrm{c}^{23} / \mathrm{FM} 7 \mathrm{c}$ flies were rebalanced such that the ns3 mutant chromosome (P[lacW]1(1)G0431 $1^{\mathrm{G} 0431}$ $\mathrm{w}^{67 \mathrm{c} 23}$ ) was balanced over FM7i, P[ActGFP]JMR3/C(1)DX (referred to as FM7, Act-GFP) to allow fully mutant animals to be distinguished by lack of GFP expression. For experiments on larvae, ns3 mutant/Y males were crossed with ns3 mutant/ FM7, Actin-GFP females and short egg lays were collected. Progeny emerging from this cross were separated into non-GFP "ns3 mutant" animals (meaning a mixture of ns3 mutant/ns3 mutant and ns3 mutant/Y) and GFP-positive animals that we refer to as "ns3 heterozygotes" (meaning a mixture of ns3 mutant/FM7, Actin-GFP and FM7, Actin-GFP/Y). The location of the G0431 P-element insertion was mapped by inverse PCR.

\section{DNA constructs}

pTWV, AT23067 (ns1, CG3983 EST), SD10213 (ns2, CG6501 EST), and GH06695 (ns3, CG14788 EST) were from the Drosophila Genomics Resource Center.

Sequence analysis and multiple sequence alignments Sequences were aligned using ClustalW2 and regions of homology shaded using BOXSHADE 3.21.

\section{Staging of Drosophila larvae}

Larvae were staged based on the morphology of mouth hooks (number of "teeth") and the presence and morphology of the anterior spiracles (Ashburner and Thompson 1978).

\section{Cell culture and transfections}

Drosophila $\mathrm{S}^{2} \mathrm{R}^{+}$cells were grown in Schneider's insect medium (GIBCO) with $10 \%$ fetal bovine serum and antibiotics and transfected using Effectene (Qiagen) according to the manufacturer's instructions.

Immunocytochemistry, immunohistochemistry, and microscopy

Larvae were dissected and fixed on ice in $4 \%$ paraformaldehyde, then further fixed at room temperature (RT). Cultured cells were washed with PBS, then fixed at RT in $4 \%$ paraformaldehyde. For staining with anti-serotonin and anti-DILP2 antibodies, tissue was subjected to antigen retrieval by incubation for $15 \mathrm{sec}$ in Re-Blot (Millipore) or by boiling in $10 \mathrm{mM}$ citrate buffer (pH 6.0) for $10 \mathrm{~min}$. After fixation, larval tissues or cells 
were washed in PBS with $0.1 \%$ Triton X-100, and $0.05 \%$ BSA (Wash Buffer), blocked in wash buffer additionally containing $10 \%$ normal goat serum (Block Buffer), and incubated with primary antibodies diluted in Block Buffer. Primary antibodies were diluted as follows: rabbit anti-phospho S505 dAkt (Cell Signaling) 1:250, mouse anti-Ribosomal Protein S6 (Cell Signaling) 1:100, rabbit anti-Drosophila Rab5 1:50 (a gift of Marcos Gonzalez-Gaitan, University of Geneva), mouse anti-Fibrillarin (AbCam) 1:500, mouse anti-serotonin (Spring Biosciences) 1:30, rabbit anti-DILP2 (a gift of Seung Kim, Stanford University) 1:500. Following primary antibody incubation, samples were washed extensively in Wash Buffer, and incubated with Alexaconjugated goat anti-mouse or goat anti-rabbit secondary antibodies (Molecular Probes) diluted 1:500 in Block Buffer. Samples were again washed extensively, and then counterstained with Hoechst 33342 (Molecular Probes) diluted 1:1000 in PBS. Images were acquired using a TCS SP2 confocal microscope (Leica) fitted with $40 x$ and $63 \times$ oil-immersion objectives. To quantify DILP2 levels (Fig. 7G), confocal Z-series of the IPCs were obtained using a 1-um step size and identical laser power and scan settings. Leica confocal software was used to generate maximum intensity 3D projections of the Z-stacks and then to measure mean fluorescent intensity across the IPCs.

\section{Fluorescent in situ hybridization}

Fluorescently labeled in situ probes were generated using the FISH Tag RNA Multicolor Kit (Molecular Probes), and hybridization was performed as previously described (Kosman et al. 2004). Images were acquired as described in the Microscopy section above.

\section{dsRNA production and embryo microinjection}

Approximately $500 \mathrm{bp}$ regions of the ns1, ns2, and ns3 ORFs were selected that did not contain any sequences with stretches longer than $18 \mathrm{bp}$ that perfectly matched other Drosophila genes. These regions were amplified by PCR, and flanking T3 RNA polymerase transcription sites were introduced. dsRNA was produced by in vitro transcription. One-hundred-fifty yw embryos were injected per condition with $2 \mu \mathrm{M}$ of dsRNA. At least two nonoverlapping $\sim 500$-bp regions were targeted within each gene.

\section{Generation of NS-YFP expression vectors and transgenic flies}

The ns1 (CG3983), ns2 (CG6501), and ns3 [CG14788, 1(1)G0431] ORFs were amplified by PCR from the AT23067, SD10213, and GH06695 ESTs, respectively. Flanking Gateway (Invitrogen) recombination sites were introduced. The PCR products were subcloned into pTWV using site-specific recombination according to the manufacturer's Gateway cloning protocol. Expression vectors were microinjected into yw embryos for generation of transgenic fly lines.

\section{Analysis of larval growth rate}

ns3 mutant/Y males were mated with ns3 mutant/FM7, ActinGFP females and allowed to lay on molasses-agar caps for 1-h intervals. Starting at $24 \mathrm{~h}$ after egg lay, 10 "ns3 mutant" animals (ns3 mutant/ns3 mutant or ns3 mutant/Y) or "ns3 heterozygous" animals (ns3 mutant/FM7, Actin-GFP or FM7, Actin$\mathrm{GFP} / \mathrm{Y}$ ) were randomly selected every $24 \mathrm{~h}$ and photographed using an MZ16 stereomicroscope and CCD camera (Leica). Images were analyzed using MetaMorph software (Molecular Devices) to determine the length of each animal.

\section{Weighing flies}

Flies were weighed (typically in groups of five to 40) using an analytical balance accurate to $\pm 0.01 \mathrm{mg}$ (Mettler Toledo).

\section{Analysis of cell size and number}

Wings from wild-type or ns 3 mutant males were imaged using differential interference contrast microscopy. Wing area was measured using MetaMorph software. Mean cell area was determined by counting the number of wing hairs in $40 \times 40$ - $\mu \mathrm{m}$ squares in the area between wing veins vII and vIII. Cell number was determined by dividing the total wing area of wild-type and ns3 mutant wings by the calculated cell area.

\section{Clonal analysis}

FLP/FRT mitotic recombination was used to generate genetic mosaics. The ns3 mutation was recombined onto a chromosome containing an FRT site at cytological position 18A as well as ubiquitin-driven nuclear GFP. Males harboring this recombined chromosome were crossed with females lacking the ns3 mutation or GFP, but containing an FRT site at cytological position 18A as well as a second chromosome heat-shock promoter-driven FLP. Embryos from this cross were collected from short egg lays and $\sim 60 \mathrm{~h}$ later, larvae were heat-shocked for $1 \mathrm{~h}$ at $37^{\circ} \mathrm{C}$ to induce FLP expression and generate mitotic clones. $\mathrm{TOR}^{\Delta \mathrm{P}}$ mitotic clones were generated as described previously (Zhang et al. 2000). Larvae were dissected $\sim 120 \mathrm{~h}$ after egg lay, immunostained, and imaged using a TCS SP2 confocal microscope (Leica). Mutant cell clones were marked by two copies of GFP for $n s 3$ mutant clones and lack of GFP for $\mathrm{TOR}^{\Delta \mathrm{P}}$ clones, and wild-type twin-spot cell clones were marked by lack of GFP for $n s 3$ mutant clones and two copies of GFP for TOR ${ }^{\Delta \mathrm{P}}$ clones. Clone size was measured using MetaMorph software.

\section{Rescue experiments}

yw; NS3-YFP; X-Gal4 or yw; X-Gal4; NS3-YFP males (where X is any Gal4 driver) were crossed with ns3 mutant/FM7, ActinGFP females and the flies were turned into fresh vials each day. The vials were checked every day and any male flies emerging were categorized as control (distinguished by the dominant bareye marker that indicates the presence of the $\mathrm{X}$ chromosome lacking the $n s 3$ mutation), ns3 mutant (distinguished by the lack of bar-eye and no expression of YFP), or ns3 mutant expressing the NS3-YFP transgene (distinguished by the lack of bar-eye and expression of YFP). The ratio of genotypes observed was compared with the expected Mendelian ratio to determine relative viability.

\section{shRNA expression experiments}

UAS-ns3-shRNA males (VDRC 18,379 or 18,380) or UAS-dilp2shRNA males (VDRC 44,761) were crossed with DDC-Gal4 females and the flies were turned into fresh vials each day. The vials were checked every day and any flies emerging were counted and then weighed $3 \mathrm{~d}$ later.

\section{Serotonin ELISA}

Flies were snap-frozen in liquid nitrogen and then maintained on a dry ice-cooled metal block while their heads were removed. Thirty-five heads were used per assay. The fly heads were ground into powder in the same cooled block using a disposable tissue grinding pestle, then resuspended in PBS with $0.1 \%$ 
ascorbic acid. Serotonin was subjected to acylation, then quantified using an ultrasensitive ELISA kit (IBL) according to the manufacturer's instructions.

\section{5-Hydroxy-L-tryptophan feeding experiments}

Newly hatched first instar larvae were placed on standard Drosophila medium additionally containing vehicle or 5-hydroxyL-tryptophan (Sigma) at a concentration of $0.5,1.0$, or $2.0 \mathrm{mg}$ / $\mathrm{mL}$. The vials were checked twice daily for the appearance of pupae.

\section{Quantitative real-time PCR}

mRNA was purified from groups of 30 flies using an Oligotex kit (Qiagen). cDNA was synthesized using SuperScript III (Stratagene) followed by quantitative real-time PCR using a 7300 Real-Time PCR System (ABI) and TaqMan primer/probe sets and Master Mix (ABI) for the gene of interest and for a GAPDH control. mRNA levels were compared between conditions using the $\Delta \Delta \mathrm{Ct}$ method.

\section{Preparation of larval extracts and immunoblot analysis}

Wandering third instar ns3 mutant or heterozygous larvae were snap-frozen in liquid nitrogen, then thawed rapidly and ground using a prechilled glass dounce homogenizer in ice-cold buffer containing $20 \mathrm{mM}$ Hepes (pH 7.5), $150 \mathrm{mM} \mathrm{KCl,} \mathrm{1.0 \%} \mathrm{Triton}$ $\mathrm{X}-100,1.0 \% \mathrm{NP}-40,0.1 \%$ SDS, $5 \mathrm{mM} \mathrm{MgCl}_{2}, 10 \mathrm{mM}$ EDTA, 1 mM EGTA, $1 \mathrm{mM}$ DTT, P8340 protease inhibitor cocktail (Sigma), Complete EDTA-free Protease Inhibitor cocktail (Roche), and Phosphatase Inhibitor cocktails 1 and 2 (Sigma). Homogenates were centrifuged for $5 \mathrm{~min}$ at $3000 \mathrm{~g}$ and the protein concentration of the supernatants determined by Bradford assay. Eighty micrograms of larval extract were loaded per lane on SDS-PAGE gels. Gels were transferred to nitrocellulose membranes and immunoblotted with the following primary antibodies: rabbit anti-phospho S505 dAkt (Cell Signaling) 1:500, mouse anti-Ribosomal Protein S6 (Cell Signaling) 1:200, and mouse anti-Actin (Chemicon) 1:500. Horseradish peroxidaseconjugated secondary antibodies were used followed by enhanced chemiluminescent detection.

\section{Akt1 rescue and scanning electron microscopy}

$\mathrm{y}^{1} \mathrm{w}^{1118} / \mathrm{Y}$ or $n s 3$ mutant/Y or $\mathrm{y}^{1} \mathrm{w}^{1118} / \mathrm{Y}$; GMR::Akt1 ${ }^{\mathrm{T} 342 \mathrm{D}, \mathrm{S505D}}$ or ns3 mutant/Y; GMR::Akt1 ${ }^{\mathrm{T} 342 \mathrm{D}, \mathrm{S} 505 \mathrm{D}}$ flies in $50 \%$ glycerol were snap-frozen in liquid nitrogen, heads were removed and cut in half sagittally and immediately fixed in $2 \%$ glutaraldehyde. Following fixation, heads were dehydrated in a $50 \%-100 \%$ ethanol series followed by hexamethyldisilazane treatment, desiccated under vacuum, mounted on conductive tape, sputter coated with gold, and imaged with a S-3400N variable pressure scanning electron microscope (Hitachi). Images were analyzed using MetaMorph software.

\section{Acknowledgments}

We thank Matt Fish for performing embryo microinjections, John Perrino for assistance with electron microscopy, members of the Scott and Meyer labs for many helpful suggestions, and Seung Kim and Liqun Luo for critical reading of the manuscript. Akt fly stocks were provided by Marc Dionne (King's College London). Anti-DILP2 antibody and dilp2 TaqMan primer/probe sets were provided by Seung Kim (Stanford University). AntiDRab5 antibody was provided by Marcos Gonzalez-Gaitan (University of Geneva). Ring gland Gal4 stocks were provided by Carl Thummel (University of Utah). DILP2 fly stocks were provided by Seung Kim (Stanford University). OR83B fly stocks were provided by Liqun Luo (Stanford University). NPF fly stocks were provided by Ping Shen (University of Georgia). Hugin fly stocks were provided by Michael Pankratz (Forschungszentrum Karlsruhe). Peptidergic fly stocks were provided by Paul Taghert (Washington University). Numerous neuronal Gal4 stocks were provided by Bader Al-Anzi (Caltech). RNAi fly stocks were provided by the Vienna Drosophila RNAi Center. Other fly stocks were provided by the Bloomington stock center. This work was supported by HHMI funding to M.P.S., NIH grant MH064801 to T.M., and postdoctoral fellowships from the Developmental and Neonatal Biology Training Program at Stanford University and the American Cancer Society to D.D.K. M.P.S. is an investigator of the Howard Hughes Medical Institute.

\section{References}

Arquier, N., Geminard, C., Bourouis, M., Jarretou, G., Honegger, B., Paix, A., and Leopold, P. 2008. Drosophila ALS regulates growth and metabolism through functional interaction with insulin-like peptides. Cell Metab. 7: 333-338.

Ashburner, M. and Thompson Jr., J.N. 1978. The laboratory culture of Drosophila. In The genetics and biology of Drosophila (eds. M. Ashburner and T.R.F. Wright), Vol. 2a, pp. 1-109. Academic Press, Inc., New York.

Baker, K.D. and Thummel, C.S. 2007. Diabetic larvae and obese flies-emerging studies of metabolism in Drosophila. Cell Metab. 6: 257-266.

Bohni, R., Riesgo-Escovar, J., Oldham, S., Brogiolo, W., Stocker, H., Andruss, B.F., Beckingham, K., and Hafen, E. 1999. Autonomous control of cell and organ size by CHICO, a Drosophila homolog of vertebrate IRS1-4. Cell 97: 865-875.

Bourne, H.R., Sanders, D.A., and McCormick, F. 1991. The GTPase superfamily: Conserved structure and molecular mechanism. Nature 349: 117-127.

Brand, A.H. and Perrimon, N. 1993. Targeted gene expression as a means of altering cell fates and generating dominant phenotypes. Development 118: 401-415.

Chen, C., Jack, J., and Garofalo, R.S. 1996. The Drosophila insulin receptor is required for normal growth. Endocrinology 137: 846-856.

Cherbas, L., Hu, X., Zhimulev, I., Belyaeva, E., and Cherbas, P. 2003. EcR isoforms in Drosophila: Testing tissue-specific requirements by targeted blockade and rescue. Development 130: 271-284.

Cole, S.H., Carney, G.E., McClung, C.A., Willard, S.S., Taylor, B.J., and Hirsh, J. 2005. Two functional but noncomplementing Drosophila tyrosine decarboxylase genes: Distinct roles for neural tyramine and octopamine in female fertility. $J$. Biol. Chem. 280: 14948-14955.

Colombani, J., Raisin, S., Pantalacci, S., Radimerski, T., Montagne, J., and Leopold, P. 2003. A nutrient sensor mechanism controls Drosophila growth. Cell 114: 739-749.

Conlon, I. and Raff, M. 1999. Size control in animal development. Cell 96: 235-244.

Dieguez, C. and Casanueva, F.F. 1995. Influence of metabolic substrates and obesity on growth hormone secretion. Trends Endocrinol. Metab. 6: 55-59.

Dietzl, G., Chen, D., Schnorrer, F., Su, K.C., Barinova, Y., Fellner, M., Gasser, B., Kinsey, K., Oppel, S., Scheiblauer, S., et 
al. 2007. A genome-wide transgenic RNAi library for conditional gene inactivation in Drosophila. Nature 448: 151-156.

Edgar, B.A. 2006. How flies get their size: Genetics meets physiology. Nat. Rev. Genet. 7: 907-916.

Feldman, J.M. and Lebovitz, H.E. 1970. Serotonin inhibition of in vitro insulin release from golden hamster pancreas. Endocrinology 86: 66-70.

Friggi-Grelin, F., Coulom, H., Meller, M., Gomez, D., Hirsh, J., and Birman, S. 2003. Targeted gene expression in Drosophila dopaminergic cells using regulatory sequences from tyrosine hydroxylase. J. Neurobiol. 54: 618-627.

Gagliardino, J.J., Zieher, L.M., Iturriza, F.C., Hernandez, R.E., and Rodriguez, R.R. 1971. Insulin release and glucose changes induced by serotonin. Horm. Metab. Res. 3: 145150.

Galloni, M. and Edgar, B.A. 1999. Cell-autonomous and nonautonomous growth-defective mutants of Drosophila melanogaster. Development 126: 2365-2375.

Golic, K.G. and Lindquist, S. 1989. The FLP recombinase of yeast catalyzes site-specific recombination in the Drosophila genome. Cell 59: 499-509.

Hedges, J., West, M., and Johnson, A.W. 2005. Release of the export adapter, Nmd3p, from the $60 \mathrm{~S}$ ribosomal subunit requires Rpl10p and the cytoplasmic GTPase Lsg1p. EMBO J. 24: $567-579$.

Huang, X., Suyama, K., Buchanan, J., Zhu, A.J., and Scott, M.P. 2005. A Drosophila model of the Niemann-Pick type C lysosome storage disease: dnpcla is required for molting and sterol homeostasis. Development 132: 5115-5124.

Ikeya, T., Galic, M., Belawat, P., Nairz, K., and Hafen, E. 2002. Nutrient-dependent expression of insulin-like peptides from neuroendocrine cells in the CNS contributes to growth regulation in Drosophila. Curr. Biol. 12: 1293-1300.

Kallstrom, G., Hedges, J., and Johnson, A. 2003. The putative GTPases Nog1p and Lsg1p are required for 60S ribosomal subunit biogenesis and are localized to the nucleus and cytoplasm, respectively. Mol. Cell. Biol. 23: 4344-4355.

Kenyon, C., Chang, J., Gensch, E., Rudner, A., and Tabtiang, R. 1993. A C. elegans mutant that lives twice as long as wild type. Nature 366: 461-464.

Kim, S.K. and Rulifson, E.J. 2004. Conserved mechanisms of glucose sensing and regulation by Drosophila corpora cardiaca cells. Nature 431: 316-320.

Kosman, D., Mizutani, C.M., Lemons, D., Cox, W.G., McGinnis, W., and Bier, E. 2004. Multiplex detection of RNA expression in Drosophila embryos. Science 305: 846. doi: 10.1126/science.1099247.

Lambertsson, A. 1998. The minute genes in Drosophila and their molecular functions. Adv. Genet. 38: 69-134.

Leevers, S.J., Weinkove, D., MacDougall, L.K., Hafen, E., and Waterfield, M.D. 1996. The Drosophila phosphoinositide 3-kinase Dp110 promotes cell growth. EMBO J. 15: 65846594.

Li, H., Chaney, S., Roberts, I.J., Forte, M., and Hirsh, J. 2000. Ectopic G-protein expression in dopamine and serotonin neurons blocks cocaine sensitization in Drosophila melanogaster. Curr. Biol. 10: 211-214.

Melcher, C. and Pankratz, M.J. 2005. Candidate gustatory interneurons modulating feeding behavior in the Drosophila brain. PLOS Biol. 3: e305. doi: 10.1371/journal.pbio.0030305.

Melcher, C., Bader, R., and Pankratz, M.J. 2007. Amino acids, taste circuits, and feeding behavior in Drosophila: Towards understanding the psychology of feeding in flies and man. $J$. Endocrinol. 192: 467-472.

Monastirioti, M. 1999. Biogenic amine systems in the fruit fly Drosophila melanogaster. Microsc. Res. Tech. 45: 106-121.
Morton, G.J., Cummings, D.E., Baskin, D.G., Barsh, G.S., and Schwartz, M.W. 2006. Central nervous system control of food intake and body weight. Nature 443: 289-295.

Neckameyer, W.S., Coleman, C.M., Eadie, S., and Goodwin, S.F. 2007. Compartmentalization of neuronal and peripheral serotonin synthesis in Drosophila melanogaster. Genes Brain Behav. 6: 756-769.

Ng, M., Roorda, R.D., Lima, S.Q., Zemelman, B.V., Morcillo, P., and Miesenbock, G. 2002. Transmission of olfactory information between three populations of neurons in the antennal lobe of the fly. Neuron 36: 463-474.

Oldham, S. and Hafen, E. 2003. Insulin/IGF and target of rapamycin signaling: A TOR de force in growth control. Trends Cell Biol. 13: 79-85.

Orosco, M., Rouch, C., and Gerozissis, K. 2000. Activation of hypothalamic insulin by serotonin is the primary event of the insulin-serotonin interaction involved in the control of feeding. Brain Res. 872: 64-70.

Peschke, E., Peschke, D., Hammer, T., and Csernus, V. 1997. Influence of melatonin and serotonin on glucose-stimulated insulin release from perifused rat pancreatic islets in vitro. $J$. Pineal Res. 23: 156-163.

Reiling, J.H. and Hafen, E. 2004. The hypoxia-induced paralogs Scylla and Charybdis inhibit growth by down-regulating S6K activity upstream of TSC in Drosophila. Genes \& Dev. 18: 2879-2892.

Reynaud, E.G., Andrade, M.A., Bonneau, F., Ly, T.B., Knop, M., Scheffzek, K., and Pepperkok, R. 2005. Human Lsg1 defines a family of essential GTPases that correlates with the evolution of compartmentalization. BMC Biol. 3: 21. doi: 10.1186/ 1741-7007-3-21

Rulifson, E.J., Kim, S.K., and Nusse, R. 2002. Ablation of insulin-producing neurons in flies: Growth and diabetic phenotypes. Science 296: 1118-1120.

Salvaterra, P.M. and Kitamoto, T. 2001. Drosophila cholinergic neurons and processes visualized with Gal4/UAS-GFP. Brain Res. Gene Expr. Patterns 1: 73-82.

Sanders-Bush, E. and Mayer, S.E. 2001. 5-Hydroxytryptamine (serotonin) receptor agonists and antagonists. In Goodman Gilman's: The pharmacological basis of therapeutics, 10th Ed., pp 281-284. McGraw-Hill, New York.

Sarbassov, D.D., Guertin, D.A., Ali, S.M., and Sabatini, D.M. 2005. Phosphorylation and regulation of Akt/PKB by the rictor-mTOR complex. Science 307: 1098-1101.

Schaeffer, H.J. and Sirotkin, A.V. 1997. Melatonin and serotonin regulate the release of insulin-like growth factor-I, oxytocin and progesterone by cultured human granulosa cells. Exp. Clin. Endocrinol. Diabetes 105: 109-112.

Schwartz, M.W., Woods, S.C., Porte Jr., D., Seeley, R.J., and Baskin, D.G. 2000. Central nervous system control of food intake. Nature 404: 661-671.

Siegmund, T. and Korge, G. 2001. Innervation of the ring gland of Drosophila melanogaster. J. Comp. Neurol. 431: 481-491.

Sink, H., Rehm, E.J., Richstone, L., Bulls, Y.M., and Goodman, C.S. 2001. sidestep encodes a target-derived attractant essential for motor axon guidance in Drosophila. Cell 105: 57-67.

Sykes, P.A. and Condron, B.G. 2005. Development and sensitivity to serotonin of Drosophila serotonergic varicosities in the central nervous system. Dev. Biol. 286: 207-216.

Sze, J.Y., Victor, M., Loer, C., Shi, Y., and Ruvkun, G. 2000. Food and metabolic signalling defects in a Caenorhabditis elegans serotonin-synthesis mutant. Nature 403: 560-564.

Tabata, T., Schwartz, C., Gustavson, E., Ali, Z., and Kornberg, T.B. 1995. Creating a Drosophila wing de novo, the role of engrailed, and the compartment border hypothesis. Development 121: 3359-3369. 
Taghert, P.H., Hewes, R.S., Park, J.H., O'Brien, M.A., Han, M., and Peck, M.E. 2001. Multiple amidated neuropeptides are required for normal circadian locomotor rhythms in Drosophila. J. Neurosci. 21: 6673-6686.

Telib, M., Raptis, S., Schroder, K.E., and Pfeiffer, E.F. 1968. Serotonin and insulin release in vitro. Diabetologia 4: 253-256.

Timmons, L., Becker, J., Barthmaier, P., Fyrberg, C., Shearn, A., and Fyrberg, E. 1997. Green fluorescent protein/ $\beta$-galactosidase double reporters for visualizing Drosophila gene expression patterns. Dev. Genet. 20: 338-347.

Tsai, R.Y. and McKay, R.D. 2002. A nucleolar mechanism controlling cell proliferation in stem cells and cancer cells. Genes \& Dev. 16: 2991-3003.

Valles, A.M. and White, K. 1986. Development of serotonincontaining neurons in Drosophila mutants unable to synthesize serotonin. J. Neurosci. 6: 1482-1491.

Valles, A.M. and White, K. 1988. Serotonin-containing neurons in Drosophila melanogaster: Development and distribution. J. Comp. Neurol. 268: 414-428.

Verdu, J., Buratovich, M.A., Wilder, E.L., and Birnbaum, M.J. 1999. Cell-autonomous regulation of cell and organ growth in Drosophila by Akt/PKB. Nat. Cell Biol. 1: 500-506.

Wimberly, B.T., Brodersen, D.E., Clemons Jr., W.M., MorganWarren, R.J., Carter, A.P., Vonrhein, C., Hartsch, T., and Ramakrishnan, V. 2000. Structure of the 30 S ribosomal subunit. Nature 407: 327-339.

Wu, Q., Wen, T., Lee, G., Park, J.H., Cai, H.N., and Shen, P. 2003. Developmental control of foraging and social behavior by the Drosophila neuropeptide Y-like system. Neuron 39: 147-161.

Xiong, W.C., Okano, H., Patel, N.H., Blendy, J.A., and Montell, C. 1994. repo encodes a glial-specific homeo domain protein required in the Drosophila nervous system. Genes \& Dev. 8: 981-994.

Zhang, H., Stallock, J.P., Ng, J.C., Reinhard, C., and Neufeld, T.P. 2000. Regulation of cellular growth by the Drosophila target of rapamycin dTOR. Genes \& Dev. 14: 2712-2724.

Zimmermann, G., Furlong, E.E., Suyama, K., and Scott, M.P. 2006. Mes2, a MADF-containing transcription factor essential for Drosophila development. Dev. Dyn. 235: 3387-3395.

Zinke, I., Kirchner, C., Chao, L.C., Tetzlaff, M.T., and Pankratz, M.J. 1999. Suppression of food intake and growth by amino acids in Drosophila: The role of pumpless, a fat body expressed gene with homology to vertebrate glycine cleavage system. Development 126: 5275-5284. 


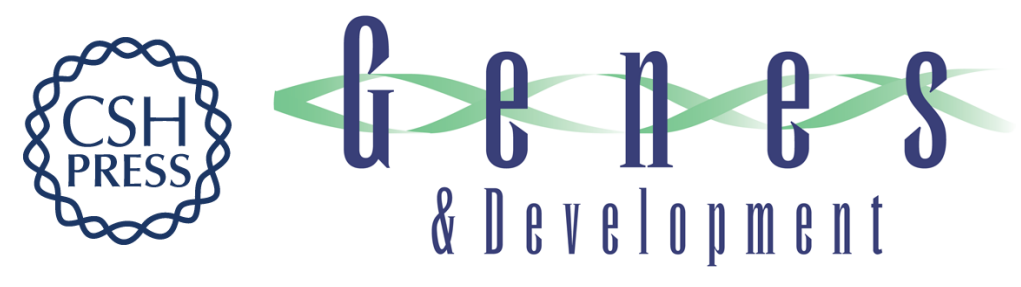

\title{
A nucleostemin family GTPase, NS3, acts in serotonergic neurons to regulate insulin signaling and control body size
}

\author{
Daniel D. Kaplan, Gregor Zimmermann, Kaye Suyama, et al.
}

Genes Dev. 2008, 22:

Access the most recent version at doi:10.1101/gad.1670508

\section{Supplemental http://genesdev.cshlp.org/content/suppl/2008/07/01/22.14.1877.DC1 \\ Material}

Related Content Serotonin and insulin signaling team up to control growth in Drosophila Anne-Françoise Ruaud and Carl S. Thummel

Genes Dev. July, 2008 22: 1851-1855

References This article cites 62 articles, 19 of which can be accessed free at: http://genesdev.cshlp.org/content/22/14/1877.full.html\#ref-list-1

Articles cited in:

http://genesdev.cshlp.org/content/22/14/1877.full.html\#related-urls

License Freely available online through the Genes \& Development Open Access option.

Email Alerting Receive free email alerts when new articles cite this article - sign up in the box at the top Service right corner of the article or click here.

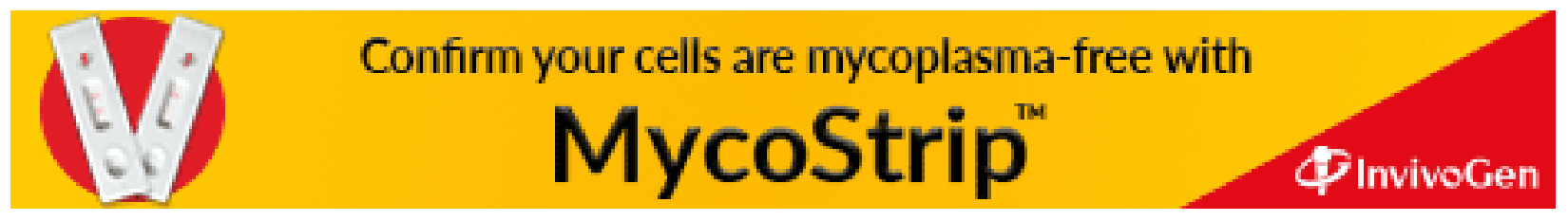

